\title{
Estructura de las comunidades de corales y octocorales de Isla de Aves, Venezuela, Caribe Nororiental
}

\author{
Yranzo. A ${ }^{1 *}$, E. Villamizar ${ }^{1}$, M. Romero \& H. Boadas ${ }^{2}$ \\ 1. Laboratorio de Ecología de Sistemas Acuáticos, Centro de Ecología y Evolución, Instituto de Zoología y Ecología \\ Tropical, Universidad Central de Venezuela, \\ 2. Museo Marino de Margarita, Boca de Rio, Venezuela; anne_YD@yahoo.com, anayranzo@gmail.com
}

$$
\text { Recibido 24-IX-2013 Corregido 08-IV-2014 Aceptado 10-IV-2014 }
$$

\begin{abstract}
Structure of the coral and octocoral communities of Isla de Aves, Venezuela, Northeast Caribbean. The Isla de Aves Wildlife Refuge is the northernmost portion of the Venezuelan territory generating $135000 \mathrm{~km}^{2}$ of Exclusive Economic Zone. Studies on coral communities are scarce and old (1970s), due to its location $650 \mathrm{~km}$ northeast of La Guaira Port and because it has military facilities. To upgrade baseline information we estimated size structure, percent live cover, species composition and abundance of corals and octocorals. We evaluated 16 sites around the island using the AGRRA Protocol (band-transects $10 \mathrm{~m}^{2}$ ) between 1.5 and $21 \mathrm{~m}$ depth ( $\mathrm{n}=67$ transects), and visual surveys conducted in other five sites. We recorded 2327 colonies belonging to four hydroid species and 36 species of stony corals in 11 families. The values for diversity, dominance and evenness of the coral community ranged between 0.78 and 2.12 (SW), 0.15 and 0.61 and 0.57 y 0.92 respectively. Most coral species had relative abundance values under 3\%, except Porites astreoides (25.57\%), Pseudodiploria strigosa (18.22\%) and Siderastrea siderea (14.44\%). They were represented mostly by smaller colonies, between 3 and $30 \mathrm{~cm}$ in maximum diameter and between 0 and $5 \mathrm{~cm}$ high. A total of 13 octocoral species belonging to three families were identified. Pseudopterogorgia americana was the most abundant species. The mean percent of live coral (including hydrocorals) was $22.30 \%$ ( $\mathrm{SE}=1.73)(15.45 \%$ for dead coral, $\mathrm{SE}=3.28$ ). Dead coral had the highest percentage of dissimilarity between sites (9.21\%) (ANOSIM) and $16.57 \%$ contribution (SIMPER analysis). Octocoral live cover ranged from 0 to $21.35 \%$ with a mean of $6.38 \%$ $(\mathrm{SE}=0.99)$. Research on benthic communities of Isla de Aves should continue, especially in the deeper areas, to assess ecological conditions. Rev. Biol. Trop. 62 (Suppl. 3): 115-136. Epub 2014 September 01.
\end{abstract}

Key words: corals, octocorals, abundance, diversity, Isla de Aves, Venezuela.

El estudio de comunidades coralinas en Venezuela se ha desarrollado especialmente en localidades como el Parque Nacional Archipiélago de Los Roques (Hung, 1985; Weil, 1985; Villamizar, Posada \& Gómez, 2003; García, Cróquer \& Pauls, 2003; Cróquer, Weil \& Zubillaga, 2005; Villamizar et al., 2008; Bastidas et al., 2012) ; el Parque Nacional Morrocoy (Villamizar, 2000; Bone et al., 2001; Cróquer \& Bone 2003; Bastidas, Cróquer \& Bone, 2006; Cróquer et al., 2010) y otras localidades del país (Olivares, 1971; Urich, 1977; Pauls, 1982; Ramírez- Villaroel, 2001; Sant, Prieto \& Méndez, 2002; Weil, 2003; Del Mónaco et al.,
2010). En contraste, las comunidades coralinas del Refugio de Fauna Silvestre Isla de Aves (RFSIA), han sido escasamente estudiadas, principalmente debido a la dificultad de acceso a la isla, ya que se encuentra a $650 \mathrm{~km}$ al noreste de la costa central de Venezuela. Esta Isla de apenas 3.72ha es el único afloramiento de la extensa cordillera submarina conocida como Prominencia de Aves y representa la porción más septentrional del territorio venezolano, generando $135000 \mathrm{~km}^{2}$ de Zona Económica Exclusiva. Su ubicación constituye un punto estratégico para Venezuela dentro de la geopolítica del Caribe en la elaboración de acuerdos 
de delimitación de áreas marinas y submarinas con los estados insulares vecinos (Arteaga, 2002) y se encuentra bajo custodia militar.

En lo que respecta a su importancia biológica - ecológica, Isla de Aves constituye el principal sitio de anidación de la tortuga verde (Chelonia mydas) en Venezuela y el segundo en el Caribe, luego de tortuguero en Costa Rica (Guada \& Solé, 2000) .Es además el área de reproducción de diversas especies de aves marinas y de descanso de numerosas aves migratorias (Phelps, 1945; Gremone \& Gómez, 1983). La importancia del Refugio de Fauna Silvestre Isla de Aves, tanto desde el punto de vista geopolítico como biológico y ecológico, motivó la realización del presente estudio, ya que el conocimiento de la diversidad de organismos marinos presentes en los fondos aledaños a Isla de Aves, fortalece la soberanía de nuestro país sobre este pequeño territorio (Arteaga, 2002). Este trabajo es el inicio de la caracterización de las comunidades bentónicas alrededor de la Isla. Únicamente Almeida and Goddard (1974) hicieron un estudio en la isla hasta los $10 \mathrm{~m}$ de profundidad, reportando 8 especies de corales e hidrocorales y 3 especies de octocorales, y Brownell y Guzmán (1974) realizaron algunas descripciones sobre los ambientes marinos en los alrededores de la Isla, enfocándose principalmente en la ictiofauna. Esta investigación tiene como objetivo actualizar y obtener una línea base cuantitativa sobre la distribución y estructura de las comunidades de corales y octocorales alrededor de la Isla.

\section{MATERIALES Y MÉTODOS}

Zona de estudio: El Refugio de Fauna

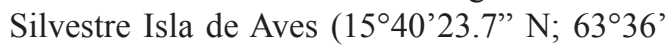
59.9 " O) se encuentra a $650 \mathrm{Km}$ al noreste del Puerto de La Guaira, y $510 \mathrm{Km}$ al Norte de la Isla de Margarita, a 200Km al Oeste de Dominica y Guadalupe y a $350 \mathrm{Km}$ al Sureste de Puerto Rico (Fig. 1). Esta Isla tiene un área

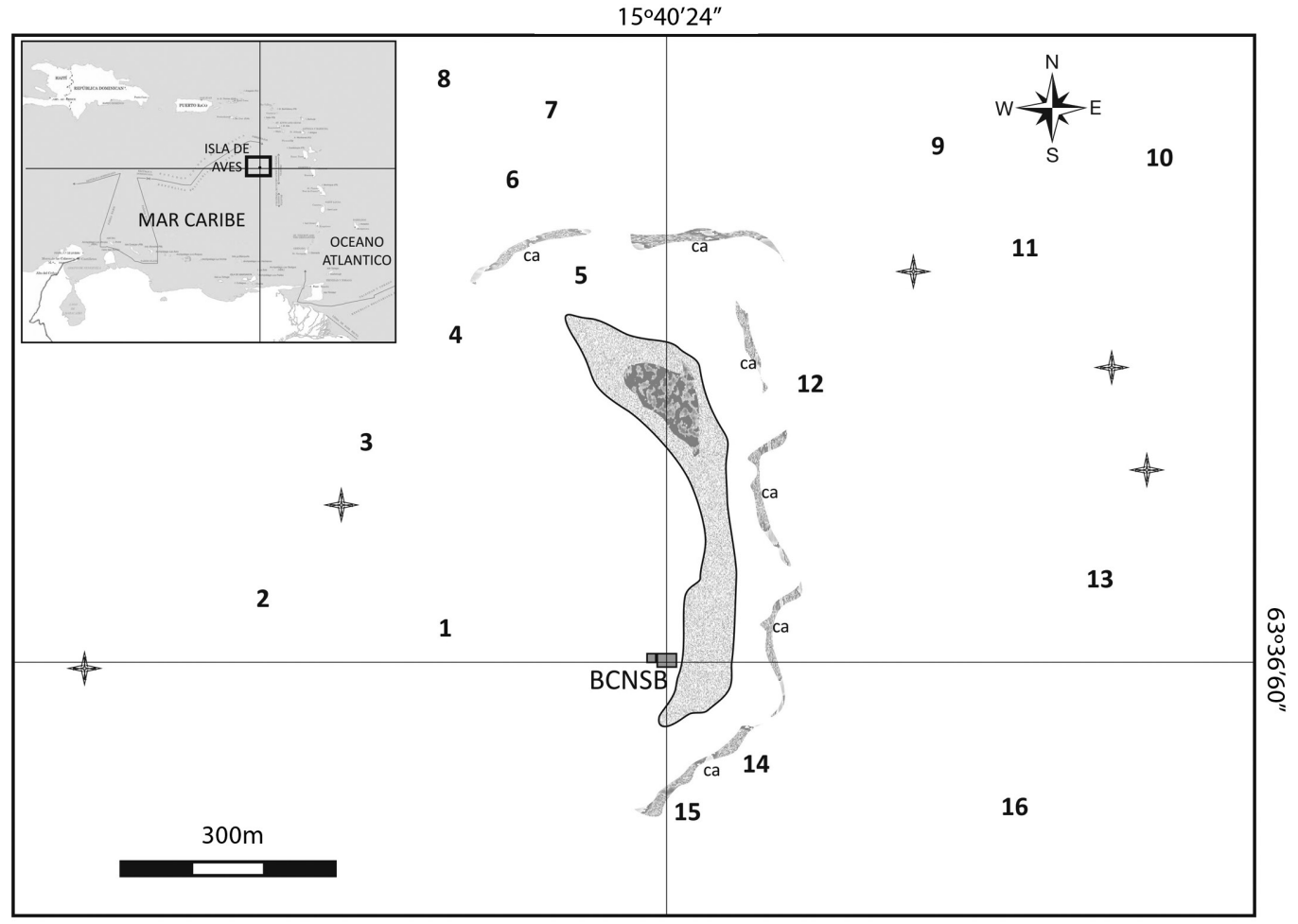

Fig. 1. El Refugio de Fauna Silvestre Isla de Aves. 
de 3.72 ha, con una altura que oscila entre 0 y $3 \mathrm{~m}$ sobre el nivel del mar y constituye el único afloramiento de la Prominencia de Aves, extensa cordillera submarina de unos $500 \mathrm{~km}$ de longitud que está conformada por un antiguo arco de islas volcánicas. Presenta una topografía plana con una superficie ligeramente ondulada por el arrastre de arena y el viento, siendo estrecha en el centro y abultada en sus extremos en dos prominencias principales que constituyen las partes más elevadas del relieve. La longitud aproximada de la isla es de $580 \mathrm{~m}$ y su ancho $130 \mathrm{~m}$ en su parte más ancha y $30 \mathrm{~m}$ en la más angosta (Arteaga, 2002). Se encuentra frecuentemente expuesta a fuertes vientos (Alisios ENE) tormentas y huracanes (Pérez Nieto, 1990), por lo que está sometida a procesos de erosión que generan cambios en su forma y dimensiones según la época del año. Particularmente en la costa central y occidental donde se presenta el seccionamiento de la isla en su parte media, durante el paso de fuertes huracanes (Pérez Nieto, 1990).Se han establecido 4 unidades litológicas a lo largo de la isla: (1) calcarenitas conformadas por fragmentos de corales, algas y gasterópodos cementados de calcita (extremos norte y sur-oeste de la isla); (2) caliza de mástil en la parte norte de la isla y en menor proporción al sur la cual es de origen coralino compuesta por caracoles, esponjas calcáreas, moluscos, arenas calcáreas y fosfáticas cementadas; (3) rocas de playa que conforman la plataforma de material clástico consolidado que rodea la isla y sobre las cuales se acumulan arenas y (4) arena de playa blanca calcárea y de granulometría media (Pantin, 1972). La única vegetación terrestre presente en el área de estudio está representada por verdolaga (Sesuvium portulacastrum y Portulaca olearacea), ambas son hierbas rastreras o levemente erguidas que cubren en forma de manto áreas de las zonas más elevadas donde desempeñan la importante función de fijar las arenas en el sector norte de la isla (Gremone \& Gómez, 1983). Al suroeste del Refugio se encuentra la Base Científico Naval Simón Bolívar, donde reside el Personal de la Armada Bolivariana de Venezuela, únicos residentes de la isla.
Se empleó el Protocolo AGGRA, utilizando bandas-transectas de $10 \mathrm{~m}^{2}$ paralelas a la costa, en las cuales se estimó la cobertura lineal (justo debajo de la cinta) y se registraron todos los corales y octocorales presentes, con el fin de determinar la composición de especies y sus respectivas abundancias. Además se estimaron las tallas tanto de corales como octocorales (diámetro máx. y altura). La cobertura lineal estimada estuvo representada por las siguientes categorías: (1) coral vivo, (2) coral muerto, (3) coral muerto con algas, (4) octocoral vivo, (5) octocoral muerto, (6) algas, (7) roca, (8) arena, (9) algas con escombros, (10) arena con escombros y (11) escombros . La categoría "coral muerto" se define como: colonias coralinas sin vida, con pocos filamentos algales dispersos a lo largo de su superficie, que al ser interceptadas por la cinta métrica, deja ver claramente la estructura esqueletal. Por otro lado, la categoría "coral muerto con algas" representa la superficie de una colonia coralina desprovista de tejido coralino y con presencia con presencia evidente (densa) de una o mas especies de algas cespitosas o macroalgas. La categoría "roca" se refiere a aquella superficie constituida por roca de playa. Según Dalongeville y Sanlaville (1984) este sustrato se define geológicamente como un depósito sedimentario con capas inclinadas hacia el mar, formado en la zona intermareal, y cuyo endurecimiento se debe a la formación de un cemento carbonático (aragonita o calcita magnesiana). Y la categoría "algas" se refiere a todo sustrato que presenta una cobertura notable de algas, no dejando ver la naturaleza del fondo.Las evaluaciones se realizaron en 16 sitios alrededor de la Isla (Fig. 1; Cuadro 1) distribuidos en 4 sectores: Sotavento, Barlovento, Norte y Sur, entre 1.5 y $21 \mathrm{~m}$ de profundidad $(n=67$ transectas). De forma complementaria se realizaron censos visuales en otros 5 sitios alrededor de la isla, con el fin de obtener el listado de especies lo más completo posible. Este estudio comprende los muestreos realizados en las Campañas de Investigación Científica organizadas anualmente (desde el 2009 al 2012) por la Dirección de Hidrografía y Navegación (DHN), durante los meses de julio y agosto. 


\section{CUADRO 1}

Sitios de muestreo en el Refugio de Fauna Isla de Aves, Venezuela

TABLE 1

Survey Sites at Isla de Aves Wildlife Refuge, Venezuela

\begin{tabular}{|c|c|c|c|c|c|c|c|}
\hline Sitio & Ubicación & $\begin{array}{l}\text { Prof. } \\
\text { (m) }\end{array}$ & $\begin{array}{l}\text { Exposión } \\
\text { al oleaje }\end{array}$ & $\begin{array}{c}\text { Intensidad } \\
\text { de corrientes }\end{array}$ & $\begin{array}{c}\text { Tipo } \\
\text { de fondo }\end{array}$ & Características Generales & $\begin{array}{c}\mathrm{N}^{\circ} \\
\text { transectas }\end{array}$ \\
\hline 1 & $\begin{array}{l}\text { Sotavento - } \\
\text { Suroeste }\end{array}$ & 6.6 & 0 & 2 & Homogéneo & $\begin{array}{l}\text { Fondo plano con roca de playa } \\
\text { intercalado con áreas arenosas- } \\
\text { Predominancia de corales del } \\
\text { género Pseudodiploria y el octocoral } \\
\text { Pseudopterogorgia americana. }\end{array}$ & 2 \\
\hline 2 & $\begin{array}{l}\text { Sotavento - } \\
\text { Suroeste }\end{array}$ & 9 & 0 & 2 & Heterogéneo & $\begin{array}{l}\text { Fondo plano con roca de playa } \\
\text { intercalado con áreas arenosas- Ligera } \\
\text { pendiente que alcanza los } 15 \mathrm{~m} \text { de } \\
\text { profundidad aprox. }\end{array}$ & 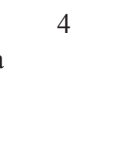 \\
\hline 3 & $\begin{array}{l}\text { Sotavento - } \\
\text { Oeste }\end{array}$ & 10 & 0 & 2 & Homogéneo & $\begin{array}{l}\text { Fondo plano con roca de playa } \\
\text { intercalado con áreas arenosas, } \\
\text { corales y esponjas en parches }\end{array}$ & 6 \\
\hline 4 & $\begin{array}{l}\text { Sotavento - } \\
\text { Noroeste }\end{array}$ & 9 & 1 & 2 & Homogéneo & $\begin{array}{l}\text { Pequeños parches rocosos } \\
\text { colonizados por corales, esponjas y } \\
\text { octocorales. Presencia de Acropora } \\
\text { palmata. }\end{array}$ & 4 \\
\hline 5 & Norte & 1.5 & 4 & 3 & Homogéneo & $\begin{array}{l}\text { Laguna con predominancia del alga } \\
\text { calcárea Lithothamium y corales } \\
\text { del género Porites: P.astreoides y } \\
\text { P.porites.Hacia el área más externa } \\
\text { de la laguna se encuentran colonias } \\
\text { muertas del género Montastraea de } \\
\text { grandes dimensiones ,colonizadas } \\
\text { principalmente por especies del } \\
\text { género Agaricia y Porites.Presencia } \\
\text { de Acropora palmata. }\end{array}$ & 6 \\
\hline 6 & Norte & $8-10$ & 0 & 3 & Homogéneo & $\begin{array}{l}\text { Fondo plano arenoso con colonias } \\
\text { de corales, octocorales y esponjas } \\
\text { distribuidas en parches }\end{array}$ & 6 \\
\hline 7 & Norte & 11 & 0 & $2 / 3$ & Homogéneo & $\begin{array}{l}\text { Fondo con pendiente leve }\left(<10^{\circ}\right) \text { en } \\
\text { dirección mar afuera. Presenta canales } \\
\text { de arena y zonas con gran abundancia } \\
\text { de macroalgas (único sitio con esta } \\
\text { característica). }\end{array}$ & 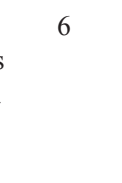 \\
\hline 8 & Noroeste & $14-15$ & 0 & 2 & Heterogéneo & $\begin{array}{l}\text { Unico sitio con estructura física } \\
\text { de carbonato de calcio (arrecife } \\
\text { consolidado) y con una pendiente que } \\
\text { alcanza los } 26 \mathrm{~m} \text { de prof. donde se } \\
\text { encuentra el quiebre hacia el talúd } \\
\text { vertical que llega hasta los } 70 \mathrm{~m} \text { y } \\
\text { luego desciende abruptamente hasta }> \\
\text { de } 100 \mathrm{~m} \text {. Esta zona profunda forma } \\
\text { parte de un halo de arena que rodea } \\
\text { a la Isla. }\end{array}$ & 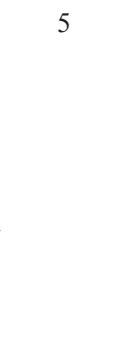 \\
\hline 9 & $\begin{array}{l}\text { Barlovento- } \\
\text { Noreste }\end{array}$ & 15 & 0 & 3 & Heterogéneo & $\begin{array}{l}\text { Fondo de roca plano cubierto por } \\
\text { corales aislados. Esponjas y gran } \\
\text { abundancia de octocorales. }\end{array}$ & 2 \\
\hline
\end{tabular}


CUADRO 1 (Continuación) / TABLE 1 (Continued)

\begin{tabular}{|c|c|c|c|c|c|c|c|}
\hline Sitio & Ubicación & $\begin{array}{l}\text { Prof. } \\
(\mathrm{m})\end{array}$ & $\begin{array}{l}\text { Exposión } \\
\text { al oleaje }\end{array}$ & $\begin{array}{c}\text { Intensidad } \\
\text { de corrientes }\end{array}$ & $\begin{array}{c}\text { Tipo } \\
\text { de fondo }\end{array}$ & Características Generales & $\begin{array}{c}\mathrm{N}^{\circ} \\
\text { transectas }\end{array}$ \\
\hline 10 & $\begin{array}{l}\text { Barlovento- } \\
\text { Noreste }\end{array}$ & 21 & 0 & 4 & Heterogéneo & $\begin{array}{l}\text { Fondo plano arenoso con colonias } \\
\text { de corales, octocorales y esponjas } \\
\text { distribuidas en parches }\end{array}$ & 2 \\
\hline 11 & $\begin{array}{l}\text { Barlovento- } \\
\text { Noreste }\end{array}$ & $17-18$ & 0 & 3 & Heterogéneo & $\begin{array}{l}\text { Fondo plano arenoso con colonias } \\
\text { de corales, octocorales y esponjas } \\
\text { distribuidas en parches }\end{array}$ & 3 \\
\hline 12 & $\begin{array}{l}\text { Barlovento- } \\
\text { Noreste }\end{array}$ & 5 & 4 & 3 & Homogéneo & $\begin{array}{l}\text { Predominancia del hidrocoral } \\
\text { Millepora squarrosa y roca de playa } \\
\text { en algunas zonas }\end{array}$ & 6 \\
\hline 13 & $\begin{array}{l}\text { Barlovento- } \\
\text { Sureste }\end{array}$ & 16 & 0 & 3 & Heterogéneo & $\begin{array}{l}\text { Fondo plano arenoso con colonias } \\
\text { de corales, octocorales y esponjas } \\
\text { distribuidas en parches }\end{array}$ & 2 \\
\hline 14 & Sur & 8 & 3 & 2 & Homogéneo & $\begin{array}{l}\text { Fondo plano con roca de playa } \\
\text { intercalado con áreas arenosas. } \\
\text { Presencia de Acropora palmata. }\end{array}$ & 6 \\
\hline 15 & Sur & 9 & 1 & 3 & Homogéneo & $\begin{array}{l}\text { Grandes rocas de playa (algunas } \\
\text { entre } 1 \text { y } 2 \text { m de altura aprox). } \\
\text { Presencia de Acropora palmata }\end{array}$ & 6 \\
\hline 16 & $\begin{array}{l}\text { Barlovento- } \\
\text { Sureste }\end{array}$ & $12-14$ & 0 & $2 / 3$ & Heterogéneo & $\begin{array}{l}\text { Fondo espacioso con colonias de } \\
\text { octocorales, esponjas y corales } \\
\text { aislados.Presencia de sustratos } \\
\text { coralinos antiguos recubiertos por } \\
\text { esponjas. }\end{array}$ & 1 \\
\hline
\end{tabular}

Nota: 6 transectas/estación. La diferencia en el esfuerzo de muestreo de algunas localidades obedece a razones logísticas .Categorías Oleaje y Corrientes: $0=$ sin impacto; $1=$ leve; $2=$ moderado; $3=$ Alto.

Note: 6 transects/site. Differences in sampling effort in some of the sites are due to logistical reasons. Waves and currents categories: $0=$ nonimpact; $1=$ slight; $2=$ moderate $3=$ high.

Las especies de corales y octocorales fueron identificadas in situ y cuando se dificultó su identificación se tomaron pequeños fragmentos de los mismos para su identificación en el laboratorio. Para la clasificación de los corales se siguió principalmente la nomenclatura de Wells (1973), Humann y DeLoach (2002), Cairns y Kitahara (2012), e incorporó la reubicación de algunas especies de la familia Agaricidae según Stemman (1991) en el género Undaria y el estudio de Budd, Fukami, Smith y Knowlton (2012) para la reubicación de varias especies. Asimismo se consideró la lista de especies de Cairns, Hoeksema y Van Der Land (1999) sobre corales extintos y corales sobrevivientes, como otro medio de corroborar la validez de los corales pétreos reportados en este estudio. En el caso de los octocorales se empleó la clave de Bayer (1961) y la información de Bayer, Grasshoff \& Verseveldt, 1983 y Janes y Wah (2005).
Análisis de datos: Se hicieron dos Análisis de Ordenamiento MDS (Índice Bray-Curtis) con el fin de explorar posibles agrupamientos entre sitios de muestreo, un MDS de acuerdo a su similaridad en la abundancia y composición de las diferentes especies coralinas y otro según la cobertura lineal de las distintas categorías bentónicas. Con el propósito de comparar la abundancia y composición de especies, así como la cobertura lineal entre sitios, se utilizó el Análisis de Similaridades (ANOSIM) y el análisis de Porcentaje de Similaridades (SIMPER); este último con el fin de cuantificar la contribución de las categorías bentónicas y de la abundancia de las distintas especies en las diferencias observadas. En ambos análisis se usó el índice de Bray - Curtis. Por otro lado, se determinaron los índices de diversidad de Shannon-Wiener (H'), dominancia de Simpson (D) y equitatividad de Pielou (J) tanto de la 
comunidad de corales como de octocorales. Todos los análisis se realizaron con el Programa estadístico PAST.

\section{RESULTADOS}

Abundancia, composición de especies y diversidad: Se registraron un total de 2327 colonias de corales e hidrocorales vivas pertenecientes a 365 especies de corales pétreos, en 11 familias y 4 especies de hidrozoos (Cuadro 2). Considerando la suma de colonias coralinas para el total de sitios evaluados, se estimó que la mayor parte de las especies de coral presentaron valores de abundancia relativa menores al 3\% (Fig. 2), a excepción de Porites astreoides (25.57\%), Pseudodiploria strigosa (18.22\%) y Siderastrea siderea (14.44\%). Dos especies del género Millepora le siguieron en abundancia: Millepora squarrosa (10.31\%) y $M$. alcicornis $(4.30 \%)$. Tanto P. astreoides como $S$. siderea estuvieron presentes en todos los sitios de muestreo (Cuadro 3) y P.strigosa en 15 de los 16 sitios. De hecho se hallaron diferencias estadísticamente significativas en composición y abundancia de las especies entre sitios (ANOSIM: $\mathrm{R}$ global $=0.657 \mathrm{p}<0.001$ ) siendo P.astreoides la especie de mayor porcentaje de disimilaridad (6.884\%) contribuyendo con $11.45 \%$ a las diferencias entre los sitios (Análisis SIMPER). La única otra especie que estuvo presente en todos los sitios de muestreo fue el hidrocoral M. alcicornis. Entre las especies del género Orbicella, O.faveolata se encontró en 10 sitios y $O$. annularis únicamente en tres. M. cavernosa presentó una mayor abundancia (3.91\%) y se encontró en 14 de los 16 sitios de muestreo. Entre los otros géneros registrados, es importante destacar la presencia del género Acropora, representado únicamente por la especie Acropora palmata, y sólo en 4 sitios de muestreo.

El número de especies de corales escleractinidos registrados en las transectas osciló

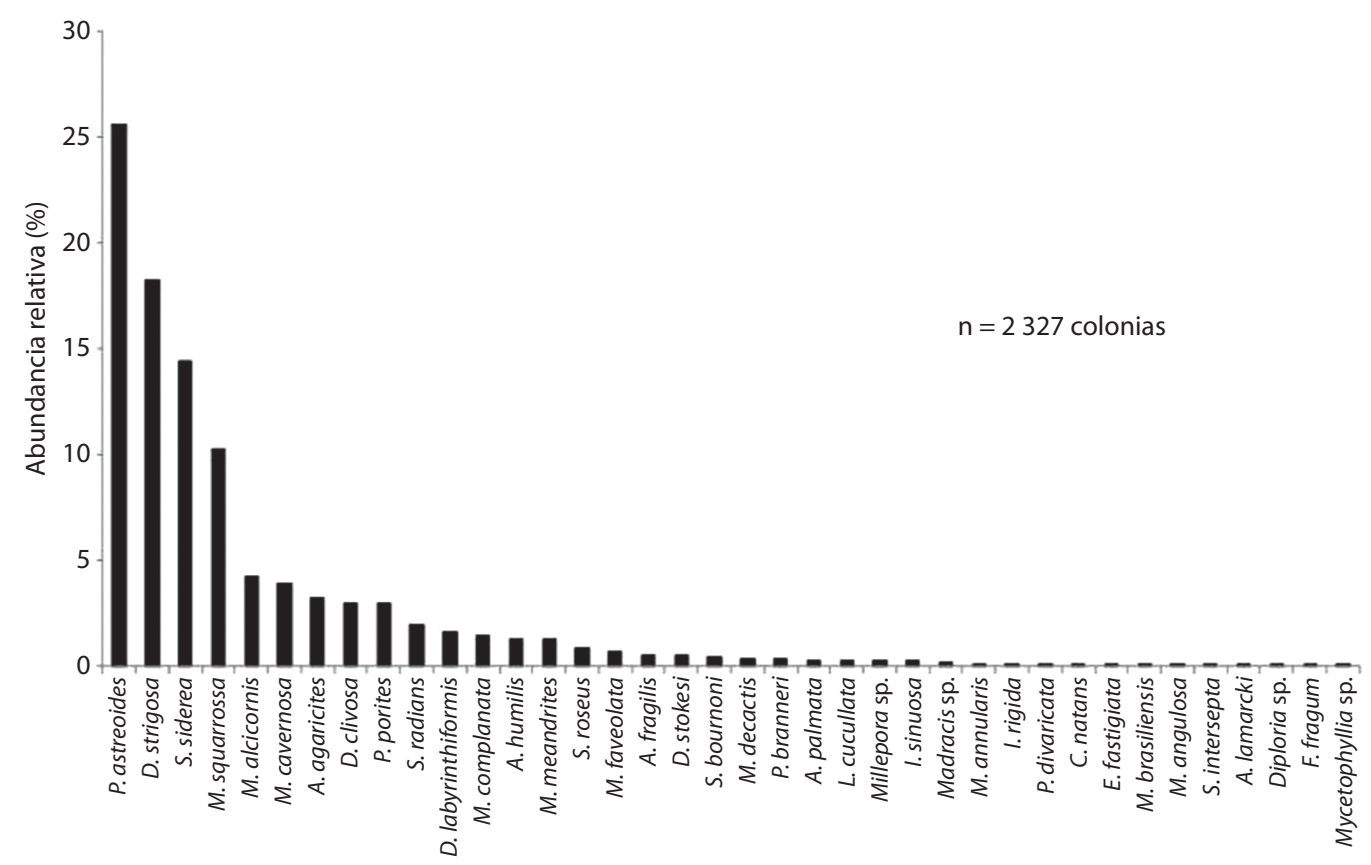

Fig. 2. Abundancia relativa de corales pétreos e hidrocorales en el Refugio de Fauna Silvestre Isla de Aves, Venezuela. Se incluyen 16 estaciones de muestreo; $n=67$ transectas

Fig. 2. Relative abundance of corals and hydrocorals in the Isla de Aves Wildlife Refuge, Venezuela. Includes 16 sampling station, $\mathrm{n}=67$ transects. 
CUADRO 2

Lista taxonómica de corales pétreos e hidrocorales del Refugio de Fauna Silvestre Isla de Aves, Venezuela

TABLE 2

Taxonomic list of hard corals and hidrocorals from Isla de Aves Wildlife Refuge, Venezuela

\begin{tabular}{|c|c|c|c|}
\hline ORDEN & SUBORDEN & FAMILIA & ESPECIE \\
\hline & ASTROCOENIIDA & Acroporidae & Acropora palmata (Lamarck,1816) \\
\hline \multirow[t]{35}{*}{ SCLERACTINIA } & FUNGIIDAE & Agariciidae & Agaricia fragilis Dana, 1846 \\
\hline & & & Agaricia lamarcki M. Edwards \& Haime, 1851 \\
\hline & & & Leptoseris cucullata (Ellis \& Solander, 1786) \\
\hline & & & Leptoseris sp. Milne Edwards \& Haime, 1849 \\
\hline & & Merulinidae & Orbicella annularis (Ellis \& Solander) \\
\hline & & & Orbicella faveolata (Ellis \& Solander, 1786) \\
\hline & & Montastraeidae & Montastraea cavernosa (Linnaeus, 1767) \\
\hline & & Undaria & Undaria agaricites Stemman,1991 \\
\hline & & & Undaria humilis Stemman,1991 \\
\hline & & Poritidae & Porites astreoides Lamarck, 1816 \\
\hline & & & Porites porites (Pallas, 1766) \\
\hline & & & Porites divaricata Le Sueur, 1821 \\
\hline & & & Porites branneri Rathbun, 1888 \\
\hline & & Siderastreidae & Siderastrea radians (Pallas, 1766) \\
\hline & & & Siderastrea siderea (Ellis \& Solander, 1786) \\
\hline & FAVIINA & Mussidae & Pseudodiploria clivosa (Ellis \& Solander, 1786) \\
\hline & & & Pseudodiploria strigosa (Dana, 1846) \\
\hline & & & Diploria labyrinthiformis (Linnaeus, 1758) \\
\hline & & & Colpophyllia natans (Houttuyn, 1772) \\
\hline & & & Favia fragum (Esper, 1795) \\
\hline & & & Manicina areolata (Linnaeus, 1758) \\
\hline & & & Solenastrea bournoni M. Edwards \& Haime, 1850 \\
\hline & & & Mussa angulosa (Pallas, 1766) \\
\hline & & & Isophyllia sinuosa (Ellis \& Solander, 1786) \\
\hline & & & Isophyllastrea rigida (Dana, 1846) \\
\hline & & & Mycetophyllia aliciae Wells, 1973 \\
\hline & & Meandrinidae & Meandrina meandrites (Linnaeus, 1758) \\
\hline & & & Meandrina danae Milne-Edwards and Haime, 1848 \\
\hline & & & Dichocoenia stokesi M. Edwards \& Haime, 1848 \\
\hline & & & Eusmilia fastigiata (Pallas, 1766) \\
\hline & & & Dendrogyra cilindrus Ehrenberg, 1834 \\
\hline & & Pocilloporidae & Madracis decactis (Lyman, 1859) \\
\hline & & & Madracis auretenra Locke, Weil \& Coates, 2007 \\
\hline & & Astrocoeniidae & Stephanocoenia intersepta (Lamarck, 1816) \\
\hline & & Rhizangiidae & Astrangia sp. Milne-Edwards \& Haime, 1848 \\
\hline \multirow[t]{4}{*}{ ANTHOATHECATA } & CAPITATA & Milleporidae & Millepora squarrosa Lamarck, 1816 \\
\hline & & & Millepora alcicornis Linnaeus, I758 \\
\hline & & & Millepora complanata Lamarck, 1816 \\
\hline & & & Stylaster roseus (Pallas, 1766 \\
\hline
\end{tabular}


CUADRO 3

Especies de corales pétreos e hidrocorales e índices de dominancia,

diversidad y equitatividad por sitio de muestreo en el Refugio de Fauna Silvestre Isla de Aves, Venezuela

TABLE 3

Hard corals and hidrocorals species and diversity, dominance and evenness index per sampling site at the Isla de Aves Wildlife Refuge, Venezuela

\begin{tabular}{|c|c|c|c|c|c|c|c|c|c|c|c|c|c|c|c|c|}
\hline ESPECIE & 1 & 2 & 3 & 4 & 5 & 6 & 7 & 8 & 9 & 10 & 11 & 12 & 13 & 14 & 15 & 16 \\
\hline A. palmata & & & & $\mathrm{x}$ & & & & & & & & $\mathrm{x}$ & & $\mathrm{x}$ & $\mathrm{x}$ & \\
\hline$U$. agaricites & & & & $\mathrm{x}$ & $\mathrm{xx}$ & & $\mathrm{x}$ & $\mathrm{x}$ & & $\mathrm{x}$ & $\mathrm{x}$ & $\mathrm{x}$ & & $\mathrm{xx}$ & $\mathrm{x}$ & \\
\hline U. humilis & $\mathrm{x}$ & & $\mathrm{x}$ & $\mathrm{x}$ & & $\mathrm{xx}$ & & & & & & $\mathrm{xx}$ & & $\mathrm{x}$ & $\mathrm{x}$ & \\
\hline A. fragilis & & & & & $\mathrm{xx}$ & $\mathrm{x}$ & & & & & & & & & $\mathrm{x}$ & \\
\hline A. lamarcki & & & & & & & & $\mathrm{x}$ & & & & & & & & \\
\hline Agaricia sp. & & & $\mathrm{x}$ & & & & & $\mathrm{x}$ & & $\mathrm{x}$ & $\mathrm{x}$ & $\mathrm{xx}$ & & $\mathrm{x}$ & $\mathrm{xx}$ & \\
\hline P. clivosa & $\mathrm{x}$ & & $\mathrm{x}$ & $\mathrm{xx}$ & $\mathrm{x}$ & $\mathrm{xxx}$ & & $\mathrm{x}$ & & & & $\mathrm{x}$ & & $\mathrm{xxx}$ & $\mathrm{xx}$ & $\mathrm{x}$ \\
\hline P. strigosa & $\mathrm{xxx}$ & $\mathrm{xxx}$ & $\mathrm{xxx}$ & $\mathrm{xxx}$ & & $\mathrm{xxx}$ & $\mathrm{xxx}$ & $\mathrm{xx}$ & $\mathrm{xx}$ & $\mathrm{x}$ & $\mathrm{x}$ & $\mathrm{x}$ & $\mathrm{xxx}$ & $\mathrm{xxx}$ & $\mathrm{xxx}$ & $\mathrm{xx}$ \\
\hline D. labyrinthiformis & & $\mathrm{xx}$ & $\mathrm{xx}$ & $\mathrm{xx}$ & & & $\mathrm{xx}$ & $\mathrm{x}$ & & & $\mathrm{x}$ & & $\mathrm{x}$ & & $\mathrm{x}$ & $\mathrm{x}$ \\
\hline Pseudodiploria sp. & & & & & & & & & & & & & & & & $\mathrm{x}$ \\
\hline D. stokesi & & $\mathrm{x}$ & & & & $\mathrm{x}$ & $\mathrm{x}$ & $\mathrm{x}$ & & & & $\mathrm{x}$ & $\mathrm{x}$ & & & \\
\hline C. natans & & & $\mathrm{x}$ & & & & & $\mathrm{x}$ & & & & & & & & \\
\hline F. fragum & & & & & & & $\mathrm{x}$ & & & & & & & & & \\
\hline E. fastigiata & & & & & & & & & & $\mathrm{x}$ & $\mathrm{x}$ & & & & & \\
\hline L. cucullata & & & & & & & & & & $\mathrm{xx}$ & & & & & $\mathrm{x}$ & \\
\hline M. alcicornis & $\mathrm{x}$ & $\mathrm{x}$ & $\mathrm{x}$ & $\mathrm{xx}$ & $\mathrm{x}$ & $\mathrm{xx}$ & $\mathrm{xx}$ & $\mathrm{xx}$ & $\mathrm{x}$ & $\mathrm{xx}$ & $\mathrm{xx}$ & $\mathrm{xx}$ & $\mathrm{x}$ & $\mathrm{x}$ & $\mathrm{x}$ & $\mathrm{x}$ \\
\hline M. complanata & & $\mathrm{x}$ & $\mathrm{xx}$ & & $\mathrm{xx}$ & $\mathrm{x}$ & & & & & & $\mathrm{xx}$ & & $\mathrm{x}$ & & \\
\hline M. squarrosa & & & & & & & & & & & & $\mathrm{xxx}$ & & $\mathrm{x}$ & & \\
\hline Millepora sp. & & & & & & & & & & & & $\mathrm{x}$ & & $\mathrm{xx}$ & & \\
\hline M. cavernosa & $\mathrm{x}$ & $\mathrm{x}$ & $\mathrm{x}$ & $\mathrm{x}$ & & $\mathrm{x}$ & $\mathrm{xx}$ & $\mathrm{xxx}$ & $\mathrm{x}$ & $\mathrm{xx}$ & $\mathrm{x}$ & $\mathrm{xxx}$ & $\mathrm{xx}$ & $\mathrm{x}$ & & $\mathrm{x}$ \\
\hline O. annularis & & & & & & & $\mathrm{x}$ & $\mathrm{x}$ & $\mathrm{x}$ & & & & & & & \\
\hline O. faveolata & & & $\mathrm{x}$ & $\mathrm{x}$ & $\mathrm{x}$ & & $\mathrm{x}$ & $\mathrm{x}$ & $\mathrm{x}$ & $\mathrm{x}$ & $\mathrm{x}$ & & $\mathrm{x}$ & $\mathrm{x}$ & & \\
\hline M. meandrites & $\mathrm{x}$ & $\mathrm{x}$ & & & & & $\mathrm{x}$ & $\mathrm{xx}$ & & $\mathrm{x}$ & $\mathrm{x}$ & & $\mathrm{x}$ & & & \\
\hline M. danae & & & & & & & & $\mathrm{x}$ & & & $\mathrm{x}$ & & & & & \\
\hline M. decactis & & $\mathrm{x}$ & $\mathrm{x}$ & & & & $\mathrm{x}$ & $\mathrm{x}$ & & $\mathrm{x}$ & & & & & & \\
\hline Madracis sp. & & & & & & & & & & & & & & $\mathrm{x}$ & $\mathrm{x}$ & \\
\hline M. aliciae & & & & & & & & $\mathrm{x}$ & & & & & & & & \\
\hline M. angulosa & & & $\mathrm{x}$ & & & & & & $\mathrm{x}$ & & & & & & & \\
\hline I. sinuosa & $\mathrm{x}$ & & & $\mathrm{x}$ & & & $\mathrm{x}$ & & & $\mathrm{x}$ & & & & & $\mathrm{x}$ & \\
\hline I. rigida & & & & & & & & & & & & & & & $\mathrm{x}$ & \\
\hline P. astreoides & $\mathrm{xx}$ & $\mathrm{xxx}$ & $\mathrm{xxx}$ & $\mathrm{xxx}$ & $\mathrm{xxx}$ & $\mathrm{xxx}$ & $\mathrm{x}$ & $\mathrm{x}$ & $\mathrm{xx}$ & $\mathrm{xx}$ & $\mathrm{xxx}$ & $\mathrm{xx}$ & $\mathrm{xxx}$ & $\mathrm{xxx}$ & $\mathrm{xxx}$ & $\mathrm{x}$ \\
\hline P. porites & $\mathrm{x}$ & & $\mathrm{x}$ & & $\mathrm{xxx}$ & $\mathrm{x}$ & & $\mathrm{x}$ & $\mathrm{x}$ & & & $\mathrm{xx}$ & $\mathrm{x}$ & $\mathrm{x}$ & & $\mathrm{x}$ \\
\hline P. branneri & & & & & & & & $\mathrm{x}$ & & & & $\mathrm{x}$ & & $\mathrm{x}$ & $\mathrm{x}$ & \\
\hline P. divaricata & & & & & & & & & & & & & & & & $\mathrm{x}$ \\
\hline S. radians & $\mathrm{x}$ & & $\mathrm{x}$ & & $\mathrm{x}$ & $\mathrm{xxx}$ & $\mathrm{x}$ & & & & & $\mathrm{x}$ & & $\mathrm{xxx}$ & $\mathrm{x}$ & \\
\hline S. siderea & $\mathrm{xx}$ & $\mathrm{xxx}$ & $\mathrm{xxx}$ & $\mathrm{x}$ & $\mathrm{x}$ & $\mathrm{xx}$ & $\mathrm{xxx}$ & $\mathrm{xxx}$ & $\mathrm{xx}$ & $\mathrm{xxx}$ & $\mathrm{xxx}$ & $\mathrm{x}$ & $\mathrm{xxx}$ & $\mathrm{xxx}$ & $\mathrm{xxx}$ & $\mathrm{xxx}$ \\
\hline S. intersepta & & & & & & & & & & & & & & $\mathrm{x}$ & $\mathrm{x}$ & \\
\hline S. bournoni & & $\mathrm{x}$ & & & & & $\mathrm{x}$ & $\mathrm{x}$ & & & & & & & & $\mathrm{x}$ \\
\hline S. roseus & & & $\mathrm{x}$ & $\mathrm{x}$ & & $\mathrm{x}$ & & $\mathrm{x}$ & & $\mathrm{x}$ & $\mathrm{xx}$ & $\mathrm{x}$ & & & $\mathrm{x}$ & \\
\hline $\mathrm{N}^{\circ}$ de especies & 11 & 11 & 17 & 13 & 10 & 13 & 16 & 22 & 9 & 14 & 13 & 18 & 10 & 19 & 19 & 11 \\
\hline $\mathrm{N}^{\circ}$ de colonias & 61 & 87 & 238 & 93 & 321 & 152 & 143 & 98 & 48 & 85 & 79 & 334 & 99 & 219 & 222 & 48 \\
\hline $\mathrm{N}^{\circ}$ de transectas & 2 & 4 & 6 & 4 & 6 & 6 & 6 & 5 & 2 & 2 & 3 & 6 & 2 & 6 & 6 & 1 \\
\hline Dominance_D & 0.26 & 0.22 & 0.25 & 0.30 & $0.61^{*}$ & 0.22 & 0.29 & $0.15^{*}$ & 0.19 & $0.15^{*}$ & 0.19 & 0.49 & 0.26 & 0.25 & 0.26 & 0.24 \\
\hline Shannon_H & 1.61 & 1.70 & 1.60 & 1.53 & $0.78^{*}$ & 1.70 & 1.51 & $2.12 *$ & 1.79 & 2.11 & 1.88 & 1.15 & 1.55 & 1.66 & 1.69 & 1.75 \\
\hline Evenness_ $\mathrm{e}^{\wedge} \mathrm{H} / \mathrm{S}$ & 0.79 & 0.85 & 0.79 & 0.79 & 0.57 & 0.88 & 0.81 & 0.88 & 0.92 & 0.88 & 0.86 & 0.57 & 0.84 & 0.83 & 0.76 & 0.82 \\
\hline
\end{tabular}

Se incluyen solo especies observadas en transectas. $x=$ rara ( 1 a 5 colonias); $x x=$ común ( 6 a 15 colonias), $x x x=a b u n d a n t e$ ( $>15$ colonias). It includes only the species observed in transects. $x=$ rare ( 1 to 5 colonies $) ; x x=$ common ( 6 to 15 colonies), $\mathrm{xxx}=$ abundant $(>15$ colonies $)$. 
entre 9 y 22 (Cuadro 3), siendo los sitios 8 y 10 , de 14.5 y $21 \mathrm{~m}$ de profundidad y situados al noroeste y noreste respectivamente, los de mayor diversidad $\left(\mathrm{H}^{\prime}=2.12\right.$ y $\left.\mathrm{H}^{\prime}=2.11\right)$ y el sitio 5, (laguna situada al norte de la isla) el de menor diversidad $\left(H^{\prime}=0.78\right)$. Por su parte, los valores de equitatividad fueron menores en los sitios 5 y 12 ( $\mathrm{J}=0.57$ para los dos sitios $)$ ambos puntos ubicados en el sector norte de la isla, con profundidades comprendidas entre 1.5 y $5 \mathrm{~m}$. El sitio 9, situado a $15 \mathrm{~m}$ de profundidad hacia Barlovento de la isla, presentó los mayores valores de equitatividad $(\mathrm{J}=0.92)$. En lo que respecta a la dominancia, esta fluctuó entre 0.15 y 0.61 , siendo el sitio 5 el de mayor dominancia y los sitios 8 y 10 los de menor dominancia (Cuadro 3).

En el caso de los octocorales (Cuadro 4), se identificaron un total de 13 especies pertenecientes a 3 familias, siendo Pseudopterogorgia americana la especie dominante. De un total de 460 colonias, P.americana presentó un $74.78 \%$ de abundancia relativa y se observó en la mayor parte de las sitios de muestreo (Cuadro 5), a diferencia de especies de otros géneros que se observaron solo en 3 sitios (ej: Eunicea sp.; E. caribaeorum). En lo que respecta a los índices de diversidad, dominancia y equitatividad para este grupo (Cuadro 5), la alta abundancia y amplia distribución de P.americana a lo largo de los sitios de muestreo, dio como resultado bajos índices de diversidad y altos valores de dominancia.

\section{Cobertura}

Se observaron 15 categorías bentónicas, de las cuales se muestran las once más representativas en el Cuadro 6. En este caso se reportan 15 sitios de muestreo, dado que no se pudo estimar la cobertura en el sitio $\mathrm{n}^{\circ} 16$. La cobertura promedio de coral vivo varió entre $8.33 \%$ ( $\mathrm{SE}=$ 2.71) en el sitio 4 (situado hacia sotavento de la isla, a $9 \mathrm{~m}$ de profundidad) y $50.50 \%(\mathrm{SE}=9.50)$ en el sitio 10 de $21 \mathrm{~m}$ de profundidad, ubicado en el sector noreste hacia Barlovento, con un promedio para todo el muestreo de $22.30 \%$ $(\mathrm{SE}=1.73)$. Mediante el Análisis de Similitud (ANOSIM; R global $=0.624 \mathrm{p}=0.001$ ) se determinó que la categoría coral muerto aportó el mayor porcentaje de disimilaridad entre sitios (9.21\%); con un porcentaje de contribución de $16.57 \%$ (Análisis SIMPER). Esta categoría bentónica presentó un promedio de $15.45 \%(\mathrm{SE}=3.28)$ y solo cuatro de las quince sitios presentaron coberturas de coral muerto

CUADRO 4

Lista taxonómica de octocorales del Refugio de Fauna Silvestre Isla de Aves, Venezuela

TABLE 4

Taxonomic list of octocorals from Isla de Aves Wildlife Refuge, Venezuela

\begin{tabular}{|c|c|c|c|}
\hline ORDEN & SUBORDEN & FAMILIA & ESPECIE \\
\hline \multirow[t]{13}{*}{ ALCYONACEA } & SCLERAXONIA & Anthotelidae & Erythropodium caribaeorum (Duchassaing \& Michelotti, 1860) \\
\hline & & Gorgonidae & Pseudopterogorgia americana (Gmelin, 1791) \\
\hline & & & Pseudoterogorgia bipinnata (Verrill, 1864) \\
\hline & & & Pseudoterogorgia sp. Kükenthal, 1919 \\
\hline & & & Pterogorgia sp. Ehrenberg, 1834 \\
\hline & & & Gorgonia ventalina (Linnaeus, 1758 ) \\
\hline & HOLAXONIA & Plexauridae & Plexaura flexuosa Lamouroux, 1821 \\
\hline & & & Plexaurella sp. Valenciennes, 1855 \\
\hline & & & Pleudoplexaura sp. Wright \& Studer, 1889 \\
\hline & & & Eunicea sp.1 Lamouroux, 1816 \\
\hline & & & Eunicea sp.2 Lamouroux, 1816 \\
\hline & & & Eunicea sp.3 Lamouroux, 1816 \\
\hline & & & Muricea sp.1 Lamouroux, 1821 \\
\hline
\end{tabular}


CUADRO 5

Especies de octocorales e índices de dominancia,diversidad y equitatividad por sitio de muestreo en el Refugio de Fauna Silvestre Isla de Aves, Venezuela

TABLE 5

Octocoral species and diversity, dominance and evenness index per sampling site at the Isla de Aves Wildlife Refuge, Venezuela

\begin{tabular}{|c|c|c|c|c|c|c|c|c|c|c|c|c|c|c|c|c|}
\hline ESPECIE & 1 & 2 & 3 & 4 & 5 & 6 & 7 & 8 & 9 & 10 & 11 & 12 & 13 & 14 & 15 & 16 \\
\hline E. caribaeorum & & & $\mathrm{x}$ & & & & & & & & & & & $\mathrm{xx}$ & $\mathrm{x}$ & \\
\hline P. americana & $\mathrm{xx}$ & $\mathrm{xxx}$ & $\mathrm{xxx}$ & $\mathrm{xxx}$ & & $\mathrm{xxx}$ & $\mathrm{xxx}$ & $\mathrm{xxx}$ & $\mathrm{x}$ & $\mathrm{xxx}$ & $\mathrm{xxx}$ & & $\mathrm{xxx}$ & $\mathrm{xx}$ & $\mathrm{xxx}$ & $\mathrm{xx}$ \\
\hline P. bipinnata & & $\mathrm{x}$ & & & & $\mathrm{x}$ & $\mathrm{x}$ & $\mathrm{x}$ & & $\mathrm{xx}$ & $\mathrm{xx}$ & & $\mathrm{x}$ & $\mathrm{xx}$ & & \\
\hline Pseudopterogorgia sp. & & $\mathrm{x}$ & & & & & $\mathrm{x}$ & $\mathrm{x}$ & & & & & & $\mathrm{x}$ & & $\mathrm{x}$ \\
\hline Pterogorgia sp. & & & & & & & & & & $\mathrm{xx}$ & $\mathrm{x}$ & & $\mathrm{xx}$ & & & $\mathrm{x}$ \\
\hline G. ventalina & & & & & & & & & & $\mathrm{x}$ & $\mathrm{x}$ & & $\mathrm{x}$ & & & \\
\hline Eunicea sp. & & & & & & $\mathrm{x}$ & & & & $\mathrm{x}$ & $\mathrm{x}$ & & & & & \\
\hline $\mathrm{N}^{\circ}$ de especies & 1 & 3 & 2 & 1 & 0 & 2 & 3 & 3 & 1 & 5 & 5 & 0 & 4 & 4 & 2 & 3 \\
\hline $\mathrm{N}^{\circ}$ de colonias & 7 & 30 & 36 & 53 & 0 & 24 & 38 & 31 & 3 & 55 & 70 & 0 & 45 & 29 & 28 & 11 \\
\hline $\mathrm{N}^{\circ}$ de transectas & 2 & 4 & 6 & 4 & 6 & 6 & 6 & 5 & 2 & 2 & 3 & 6 & 2 & 6 & 6 & 1 \\
\hline Dominancia_D & 1 & 0.73 & 0.90 & 1 & - & 0.81 & 0.86 & 0.70 & 1 & 0.39 & 0.56 & - & 0.68 & 0.86 & 0.88 & 0.61 \\
\hline Shannon_H & 0 & 0.45 & 0.14 & 0 & - & 0.29 & 0.23 & 0.47 & 0 & 1.15 & 0.79 & - & 0.55 & 0.23 & 0.17 & 0.58 \\
\hline Equitatividad_J & 0 & 0.49 & 0.20 & 0 & - & 0.41 & 0.25 & 0.68 & 0 & 0.78 & 0.62 & - & 0.50 & 0.26 & 0.19 & 0.84 \\
\hline
\end{tabular}

Se incluyen solo especies observadas en transectas. $\mathrm{x}=$ rara ( 1 a 5 colonias $) ; \mathrm{xx}=$ común $(6$ a 15 colonias $) ; \mathrm{xxx}=$ abundante ( $>15$ colonias).

Include only the species observed in transects. $\mathrm{x}=$ rare ( 1 to 5 colonies $) ; \mathrm{xx}=$ common ( 6 to 15 colonies $), \mathrm{xxx}=\mathrm{abundant}$ $(>15$ colonies $)$.

superiores al 30\%, siendo 59.75\% ( $\mathrm{SE}=11.79)$ el valor máximo (sitio $\mathrm{n}^{\circ} 3$, ubicado hacia sotavento de la isla, a 10 de profundidad). La categoría Roca, que representa el sustrato conformado por roca de playa, se registró en cinco sitios. El porcentaje de cobertura para esta categoría fue incluso superior a la de coral muerto en los sitios 1 y 5 (el primero ubicado a 6.6 metros de profundidad hacia sotavento y el segundo representado por la laguna del sector norte), lo que no es de extrañar, dado el basamento de roca que tiene la isla. Por otro lado la categoría coral muerto con algas a pesar de presentar una baja cobertura promedio, $5.06 \%$ $(\mathrm{SE}=1.50)$, se registró en varias de los sitios que poseen una baja cobertura de coral muerto.

En lo que respecta a los octocorales, se pueden destacar los sitios 4 y 8 como los de mayor cobertura lineal, con $21.35 \%(\mathrm{SE}=3.65)$ y $19.64 \%(\mathrm{SE}=3.63)$ respectivamente, siendo $6.38 \%(\mathrm{SE}=0.99)$ el valor promedio para todos los sitios. Ambos puntos de muestreo donde se registraron los mayores porcentajes de cobertura lineal (4 y 8) presentan una intensidad de corrientes moderada y se encuentran ubicados al noroeste de la isla, el primero a 9 de metros de profundidad y el segundo entre 14 y 15 metros. En dos de los sitios de muestreo (5 y 12) ambos ubicados al norte y caracterizados por fuertes corrientes y oleaje, no se registró cobertura lineal para estos organismos $\mathrm{La}$ cobertura promedio de octocoral muerto fue baja: $0.09 \%$ ( $\mathrm{SE}=0.09)$, ya que esta categoría se registró únicamente en dos de los quince sitios. Entre el resto de las categorías bentónicas se pueden destacar las algas, con una cobertura lineal promedio de $11.60 \%(\mathrm{SE}=1.80)$ siendo el sitio 7, situado al norte franco de la isla, a 11 metros de profundidad, el de mayor cobertura $(31.13 \%$; $\mathrm{SE}=4.45)$.

Estructura de tallas: Dada la importancia de los corales $P$. astreoides, $P$. strigosa y $S$. siderea y del octocoral $P$. americana, en 


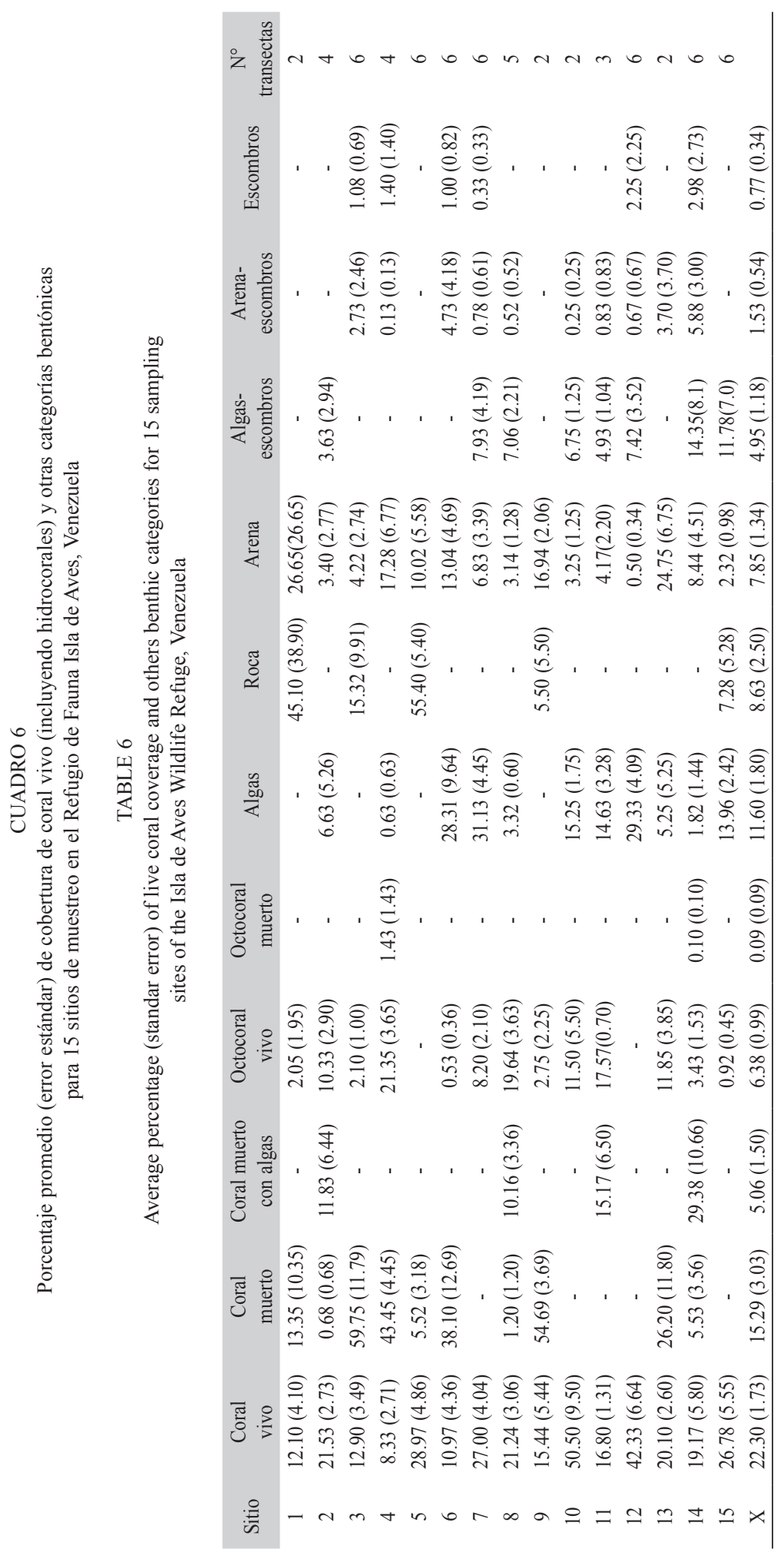


términos de su abundancia y amplia distribución a lo largo de los sitios de muestreo, a continuación se presentan las estructuras de tallas únicamente para estas especies.

En términos generales, la mayor parte de las colonias de las tres especies de corales se ubicaron en las primeras categorías de tallas, es decir las más pequeñas, tanto en su diámetro máximo como altura (Fig. 3 A y B). En el caso de P.astreoides, el $53.48 \%$ de las colonias eran pequeñas (3 a10 cm de diámetro máximo;
Fig. 3A) y bajas $(72.83 \%$ entre 0 - $5 \mathrm{~cm}$ de altura; Fig. 3B). En ambos casos (diámetro y altura) se registró una disminución en la proporción de colonias de P.astreoides hacia las tallas más grandes. En lo que respecta a P.strigosa, el $74.62 \%$ de las 327 colonias midieron entre 3 y $30 \mathrm{~cm}$ de diámetro máximo, ubicándose en las tres primeras categorías de tallas (Fig. 3A), mientras que la altura del $43.73 \%$ de las colonias no superó los $5 \mathrm{~cm}$ (Fig. 3B). Finalmente en el caso de S.siderea, la mayor parte de las
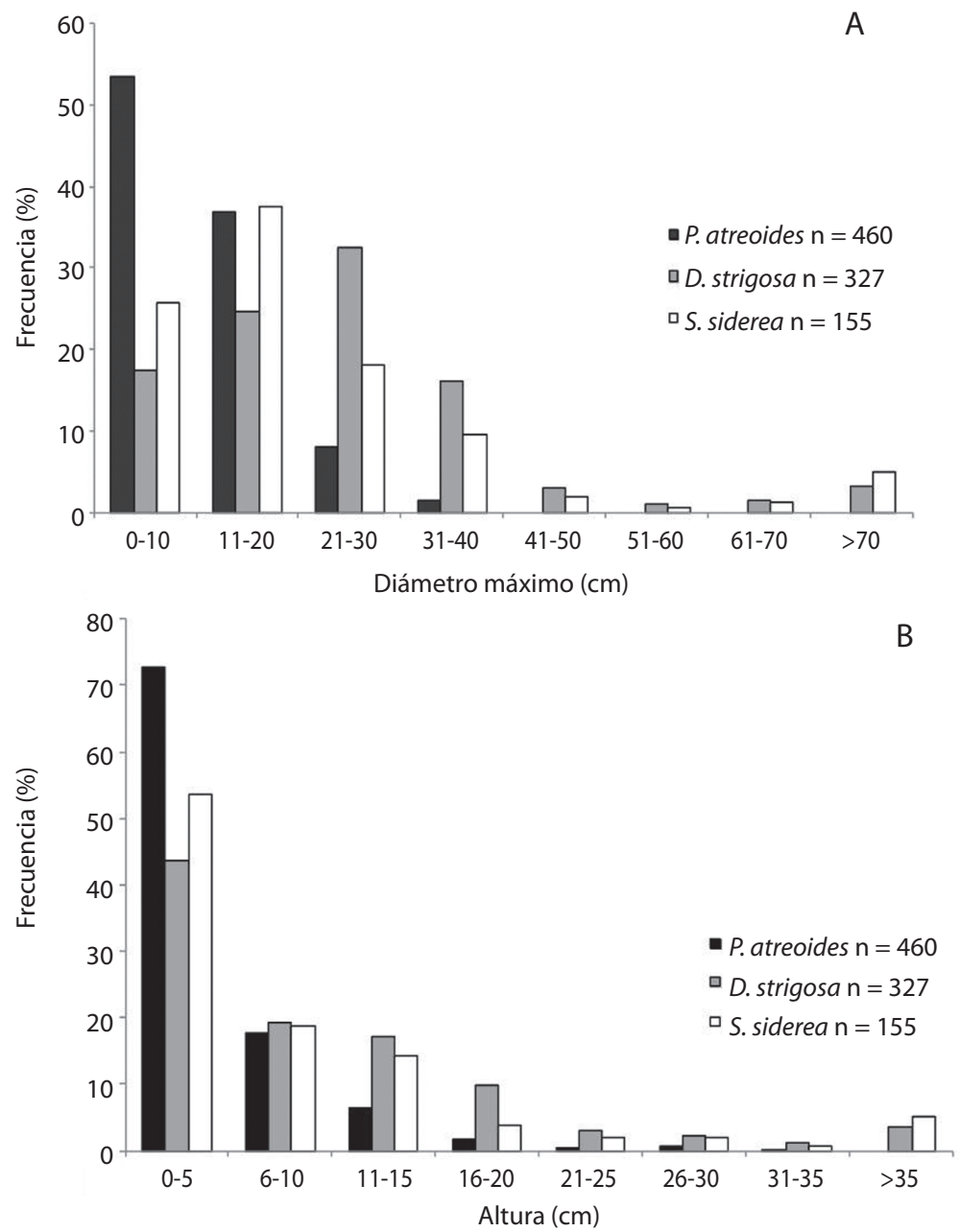

Fig. 3. Histograma de frecuencia de tallas: diámetro máximo (A) y altura (B) de las tres especies de corales pétreos de mayor abundancia en el Refugio de Fauna Silvestre Isla de Aves. Para cada especie se incluye una muestra representativa de colonias sin distinción de sitio. Diámetro mínimo incluido $=3 \mathrm{~cm}$; altura mínima incluida $=0 \mathrm{~cm}$ (colonias incrustantes). Fig. 3. Size frequency histogram: maximum diameter (A) and height (B) of the three hard coral species most abundant in the Isla de Aves Wildlife Refuge. For each species includes a representative sample of colonies regardless of site. Minimum diameter included $=3 \mathrm{~cm}$, minimum height included $=0 \mathrm{~cm}$ (encrusting colonies). 
colonias (55.48\%) presentaron entre 11 y 30 $\mathrm{cm}$ de diámetro máximo (Fig. 3A), situándose especialmente entre los 21 y $30 \mathrm{~cm}$. La altura de las colonias de esta especie (Fig. 3B) al igual que las de P.strigosa en su mayoría no superó $\operatorname{los} 5 \mathrm{~cm}(53.55 \%)$.
Una aproximación más detallada de las dimensiones de las colonias para estas especies en algunas de las localidades de muestreo, se puede observar en el Cuadro 7. Las altas desviaciones estándar que se presentan en la mayor parte de los casos, reflejan la variabilidad en

\section{CUADRO 7}

Diámetro y altura promedio $(\mathrm{cm})$ de Porites astreoides, Diploria strigosa y Siderastrea siderea en algunos sitios de muestreo en el Refugio de Fauna Silvestre Isla de Aves, Venezuela

TABLE 7

Average diameter and height $(\mathrm{cm})$ of Porites astreoides, Diploria strigosa y Siderastrea siderea in some survey sites of the Isla de Aves Wildlife Refuge, Venezuela

\begin{tabular}{|c|c|c|c|c|}
\hline Especie & Sitio & Diámetro máx. $(\mathrm{cm})$ & Altura (cm) & $\mathrm{N}^{\circ}$ de colonias \\
\hline \multirow[t]{10}{*}{ Porites astreoides } & 1 & $9.55 \pm 5.22$ & $6.36 \pm 2.34$ & 11 \\
\hline & 2 & $8.08 \pm 3.51$ & $4.78 \pm 3.95$ & 24 \\
\hline & 3 & $10.19 \pm 4.75$ & $6.04 \pm 3.03$ & 54 \\
\hline & 4 & $14.56 \pm 6.66$ & $9.82 \pm 5.76$ & 16 \\
\hline & 5 & $15.66 \pm 8.00$ & $7.73 \pm 6.03$ & 248 \\
\hline & 6 & $9.20 \pm 4.99$ & $4.9 \pm 4.00$ & 20 \\
\hline & 9 & $10.50 \pm 3.69$ & $5 \pm 0.0$ & 10 \\
\hline & 11 & $12.67 \pm 4.42$ & $4.67 \pm 4.69$ & 18 \\
\hline & 13 & $10.25 \pm 5.95$ & $4.9 \pm 1.68$ & 20 \\
\hline & 14 & $11.56 \pm 7.74$ & $3.92 \pm 5.64$ & 39 \\
\hline \multirow[t]{11}{*}{ Pseudodiploria strigosa } & 1 & $28.75 \pm 17.52$ & $14.38 \pm 12.01$ & 24 \\
\hline & 2 & $22.86 \pm 16.72$ & $9.07 \pm 9.88$ & 15 \\
\hline & 3 & $22.90 \pm 9.12$ & $9.28 \pm 6.54$ & 68 \\
\hline & 4 & $40.36 \pm 31.44$ & $18.57 \pm 20.31$ & 28 \\
\hline & 6 & $34.67 \pm 19.64$ & $14.67 \pm 13.71$ & 45 \\
\hline & 7 & $24.78 \pm 15.45$ & $10.66 \pm 10.47$ & 45 \\
\hline & 8 & $21.67 \pm 23.29$ & $13.78 \pm 16.93$ & 9 \\
\hline & 9 & $17.86 \pm 7.56$ & $6.43 \pm 3.78$ & 7 \\
\hline & 13 & $20.77 \pm 7.60$ & $5.7 \pm 2.77$ & 13 \\
\hline & 14 & $24.33 \pm 10.73$ & $9.67 \pm 9.35$ & 67 \\
\hline & 16 & $34.16 \pm 7.36$ & $9.17 \pm 7.36$ & 6 \\
\hline \multirow[t]{11}{*}{ Siderastrea siderea } & 1 & $26.82 \pm 24.42$ & $11.82 \pm 15.85$ & 11 \\
\hline & 2 & $14.5 \pm 7.96$ & $3.86 \pm 4.33$ & 14 \\
\hline & 3 & $23.20 \pm 13.92$ & $8.78 \pm 7.77$ & 50 \\
\hline & 6 & $54.62 \pm 51.94$ & $23.85 \pm 21.23$ & 13 \\
\hline & 7 & $20.83 \pm 13.58$ & $9.37 \pm 11.46$ & 41 \\
\hline & 8 & $28.89 \pm 27.78$ & $10.58 \pm 15.50$ & 19 \\
\hline & 9 & $19.62 \pm 11.98$ & $9.23 \pm 4.49$ & 13 \\
\hline & 11 & $34.62 \pm 25.94$ & $18.45 \pm 17.78$ & 13 \\
\hline & 13 & $25.71 \pm 30.55$ & $10.24 \pm 13.92$ & 31 \\
\hline & 14 & $20.50 \pm 8.13$ & $11.7 \pm 5.37$ & 30 \\
\hline & 16 & $16.76 \pm 7.28$ & $3.41 \pm 4.58$ & 17 \\
\hline
\end{tabular}

Nota: el $\mathrm{n}^{\circ}$ de colonias mostrado por sitio representa una muestra del total registrado.

Note: the number of colonies shown by site represents a sample of the total registered. 
las tallas de las colonias en un mismo sitio de muestreo, sin embargo se puede señalar que, en el caso de P.astreoides, las colonias de mayores dimensiones están en el sitio 5, el cual representa la laguna situada al norte de la isla, tanto en relación al diámetro máximo (15.66 \pm 8.00 $\mathrm{cm})$ como la altura $(7.73 \pm 6.03 \mathrm{~cm})$. Las colonias de P.strigosa presentaron sus mayores dimensiones promedio en los sitios 4 y 6 , el primero ubicado al Noroeste, hacia sotavento de la isla, a $9 \mathrm{~m}$ de profundidad caracterizado por corrientes de intensidad moderada y el segundo situado al norte franco, entre 8 y 10 metros de profundidad, con alta intensidad de corrientes. En lo que respecta a S.siderea, se repite el sitio 6 como el de colonias con las mayores dimensiones.

Por otro lado, en lo que respecta al octocoral P. americana (Fig. 4 A y B), el diámetro máximo del $73 \%$ de las colonias se ubicó en las primeras tres categorías de tallas (Fig. 4A), que abarcan de 5 a $30 \mathrm{~cm}$, mientras que la altura de las colonias presentó una distribución más uniforme a lo largo de las diferentes categorías de tallas (Fig. 4B) sin predominancia de alguna en particular.
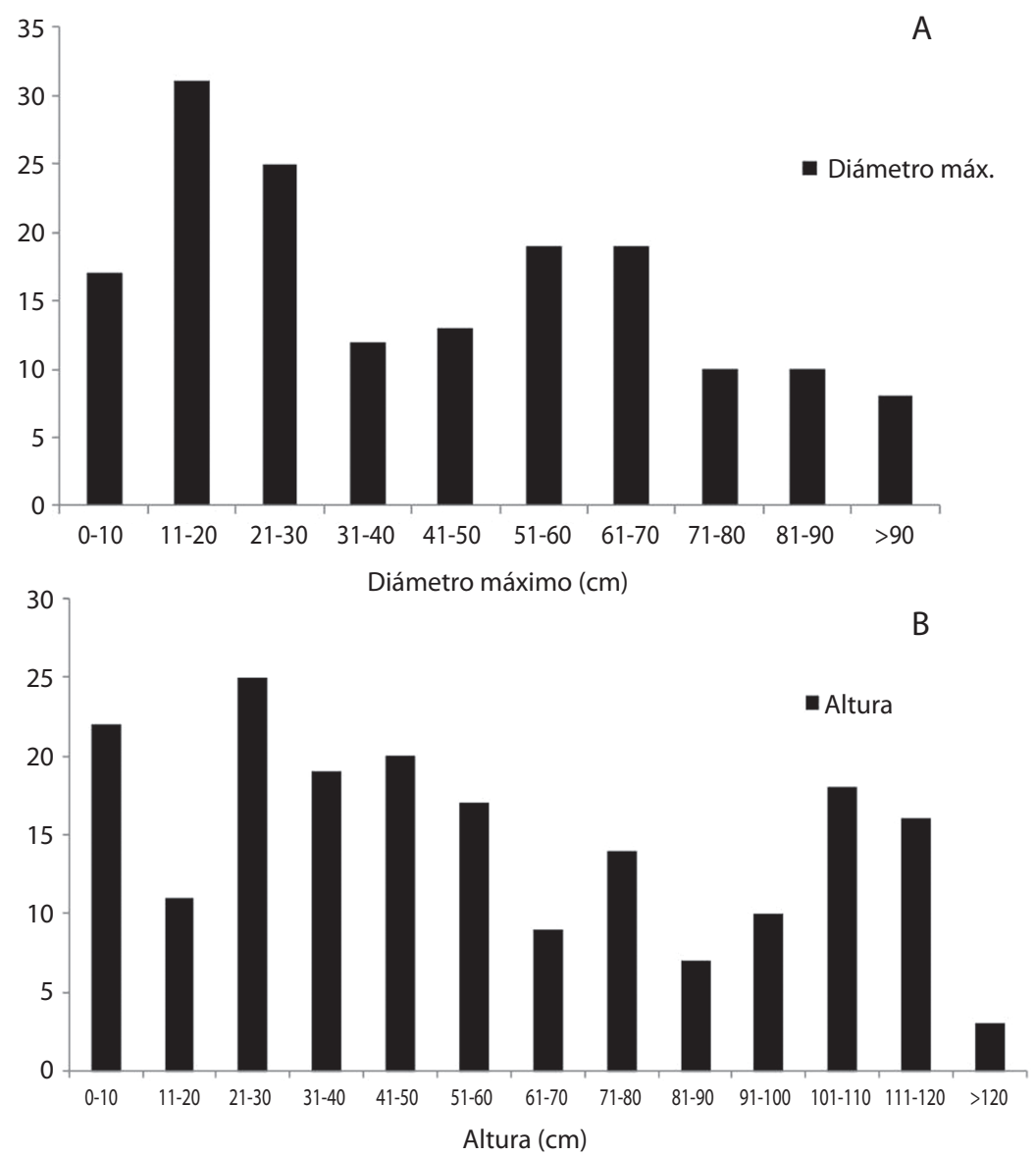

Fig. 4. Distribución de tallas (diámetro máximo y altura) del octocoral Pseudopterogorgia americana en el Refugio de Fauna Silvestre Isla de Aves

Fig. 4. Size frequency histogram (maximum diameter and height) of the octocoral Pseudopterogorgia americana in the Isla de Aves Wildlife Refuge. 


\section{DISCUSION}

Las comunidades marinas bentónicas del Refugio de Fauna Silvestre Isla de Aves, están formadas principalmente por 36 especies de corales pétreos y cuatro especies de hidrocorales. Esta riqueza es menor que la reportada para otras islas del territorio venezolano. Así, en el Parque Nacional Archipiélago de Los Roques, se han reportado 57 especies por Hung (1985); 69 (61 hermatípicos con zooxantelas y 8 ecomorfos) por Weil (2003) y 55 (Villamizar et al. en prep.). Asimismo para un arrecife de la Orchila, Urich (1977) reporta 62 especies (54 hermatípicos) y en La Blanquilla Weil (2003) encontró 47 especies (43 con zooxantelas). En otras áreas del país por el contrario, la riqueza de especies de corales es menor que la de Isla de Aves. Del Mónaco et al. (2010) registraron 23 especies en la isla de La Tortuga; Buccimazza (1984) encontró 25 para la Isla de Coche; Weil (no publicado) registró 31 especies (26 hermatípicos) en las islas de Coche y Cubagua al sur de Margarita y Boadas (2011) reportó un total de 12 especies para la isla de Los Frailes, cerca de la isla de Margarita. En relación a las Islas cercanas a la costa continental del país, en el zona occidental, en las Islas Ratón, El Rey, Isla Larga, Alcatraz y Santo Domingo, se han registrado 19 especies de corales pétreos (Rodríguez et al. 2011), Bahía de Adícora, con 13 especies (Eizaga, 2013) y en el Parque Nacional Morrocoy, 45 especies (Pauls 1995) y posteriormente, 26 especies (Villamizar 2000). En el oriente del país, del Parque Nacional Mochima se conocen 37 especies (Olivares 1971, Pauls 1982); en dos localidades del Golfo de Cariaco Sant et al. (2002) encontraron 33 especies de corales pétreos y en las Bahías de Santa Fe, Bahía de Mochima y Manzanillo, hallaron 22 especies.

Tomando como referencia el número de especies de corales (principalmente con zooxantelas y en menor extensión sin zooxantelas) en otras localidades del Caribe como Panamá (62), Costa Rica (47), República Dominicana (56), Martinica (53) y Cuba, Jamaica y Puerto Rico con 72 especies, (Miloslavich et al. 2010,
Weil, 2003, 2006; Ballantine et al., 2008; Vandel, Pichon \& Joannot, 2012), podría decirse que Isla de Aves presenta un bajo número de especies coralinas. Sin embargo, la riqueza estimada se aproxima a reportes de localidades más cercanas a la Isla hacia el este, como Guadalupe con 42 especies y Dominica con 36 y supera al número de especies reportado para otras islas como Saint Martin con 30 especies (Steiner, 2003, Vandel et al. 2012). En el caso de los octocorales, el número de especies registrado (13) se puede considerar muy bajo en relación a otros sitios como Cuba (81); Panamá (61), Antillas Holandesas (48), Republica Dominicana (44) Jamaica (43); Costa Rica y Puerto Rico (26 sp.) (Miloslavich \& Klein, 2005; Weil, 2006; Etnoyer, Wirshing \& Sánchez, 2010).

En lo que respecta a las diferencias de las comunidades de corales entre las estaciones de muestreo, los dos sitios que presentaron la mayor diversidad (sitios 8 y 10) se caracterizaron por ser zonas relativamente profundas. La estación 10, ubicada hacia Barlovento de la Isla, área caracterizada por fuertes corrientes, fue el sitio de muestreo de mayor profundidad entre todos los evaluados $(21 \mathrm{~m})$ y la estación 8 situada entre 14 y $15 \mathrm{~m}$ de profundidad, se diferenció del resto de las estaciones de muestreo por presentar un arrecife desarrollado, es decir con una estructura heterogénea de carbonato de calcio, dando lugar a un mayor número de hábitats (mayor heterogeneidad espacial) y en consecuencia a una mayor riqueza de especies. En términos generales las zonas profundas presentaron una mayor diversidad de corales que las zonas someras, a pesar de que el esfuerzo de muestreo en estos sitios ( ${ }^{\circ}$ de transectas) fue menor debido a razones logísticas.

Con relación a la equitatividad, destacan los menores valores estimados en las estaciones 5 y 12. Esto se debe a la dominancia de P.astreoides en el primer sitio y del hidrocoral $M$. squarrosa en el segundo, por lo cual los índices de diversidad de estas estaciones fueron los más bajos. Estos dos sitios fueron los más "diferentes" del resto, el primero por ser una laguna de poca profundidad, expuesta a 
constante oleaje, y el segundo, también una zona somera $(5 \mathrm{~m}$.), situado al noreste, se caracterizó por la presencia de grandes "bloques" de M. squarrosa y de roca de playa en algunas áreas. Cuando el oleaje y las corrientes son muy intensas pueden afectar el establecimiento y desarrollo de los octocorales (Kinzie, 1973; Guzmán \& Cortés, 1985) por lo cual la ausencia del octocoral dominante Pseudopterogorgia americana para estos dos únicos sitios, pudiera estar relacionada con estas variables físicas. Es posible que haya ocurrido la remoción de colonias de este octocoral por el paso de huracanes o ciclones en la zona, una posible evidencia de esto fue el encuentro anual (entre el 2009 y 2012) de numerosas bases y esqueletos de octocorales en la costa, sin embargo la ausencia de registros cuantitativos previos limitan las conclusiones que se puedan realizar al respecto.

En las comunidades coralinas de Isla de Aves las especies de corales pétreos más abundantes son P. astreoides, P.strigosa y S.siderea. Estas especies son abundantes en diversas localidades del Caribe (Guzmán \& Guevara, 2001; Bastida-Zavala, et al., 2000; Valdivia \& De la Guardia, 2004; Caballero, Rosales \& Alcalá, 2005; Weil, 2003; 2006; Lirman \& Fong, 2007; García-Sais et al., 2009; Domínguez et al., 2010) y se caracterizan por presentar una alta resistencia a diferentes factores de perturbación, entre los que se pueden mencionar la sedimentación, dada a la capacidad que tienen de eliminar partículas de sedimento acumuladas en la superficie de sus colonias ( Rogers, 1983; Sullivan, Chiappone \& Lott, 1994). S. siderea, presenta gran tolerancia a cambios de temperatura (Kemp et al., 2011) y alta plasticidad fenotípica, manifestada en cambios de morfología esqueletal de acuerdo al ambiente donde se encuentre (Foster 1979). P.astreoides, la especie de mayor abundancia relativa en el presente trabajo, se caracteriza por presentar altas tasas de fecundación y corta longevidad (Bak \& Engel, 1979; Chornesky \& Peters, 1987). Esta especie en particular se ha señalado como una de las más abundantes actualmente en arrecifes someros del Caribe, dado el incremento significativo de su abundancia en los últimos 30 años (Green, Edmunds, \& Carpenter, 2008) producto de la combinación de altas tasas de reclutamiento y tolerancia a condiciones adversas como el incremento de la temperatura, ya que poseen zooxantelas de clados resistentes a fenómenos de blanqueamiento (LaJeunesse, 2002; Lirman \& Miller, 2003; Green, et al., 2008). Las pequeñas dimensiones registradas en la mayor parte de las colonias de P.astreoides de la presente investigación, sugieren tasas de reclutamiento elevadas para esta especie en el área de estudio (Bak \& Meesters, 1999). Igualmente P. strigosa y S.siderea presentan estructuras de tallas donde predominan las colonias de tamaños reducidos, que posiblemente corresponden a colonias jóvenes. Incluso es importante acotar aunque no se presentan resultados al respecto, la observación in situ de numerosos reclutas de las tres especies durante los muestreos.

La mayor abundancia de especies como S.siderea, P.strigosa y P.astreoides reportada en diversos arrecifes de la región del Caribe, se encuentra relacionada con el deterioro progresivo que han sufrido las principales especies de corales constructores de arrecifes, lo que ha generado cambios en la composición de especies de los mismos (Green et al., 2008). En el caso de Isla de Aves, no se conocen reportes previos que puedan tomarse como referencia sobre posibles cambios en la composición de las especies dominantes, sin embargo, la presencia de grandes colonias muertas de especies como Orbicella faveolata en varias zonas en los alrededores del Refugio, especialmente en áreas profundas, así como de esqueletos de $A$. palmata y el hallazgo de restos coralinos de Acropora cervicornis en la costa, sugieren una historia similar a la de la mayor parte de los arrecifes del Caribe, donde epidemias de enfermedades (ej: Banda blanca en Género Acropora; Gladfelter 1982, Aronson \& Precht, 2001) y el cambio climático global desencadenante de fenómenos de blanqueamiento, en conjunción con la mortandad del erizo Diadema antillarum (Lessios, 1988; Carpenter, 1990) entre otros factores, han generado un deterioro progresivo 
de estas comunidades (Szmant \& Gassman, 1990; Bak \& Luckhurst, 1980; Bythell, Gladfelter \& Bythell, 1993; Bruckner \& Bruckner, 2003; Steiner \& Kerr, 2008). Es importante mencionar que aunque en muy baja proporción, se han observado en los corales algunas enfermedades en la localidad de estudio (banda negra, banda amarilla, lunares oscuros, al igual que palidez y blanqueamiento. Es posible que este resultado esté relacionado con la época del año en la cual se realizaron las expediciones al area de esudio (julio y agosto), ya que es durante los meses de septiembre y octubre cuando comienzan a evidenciarse los efectos del estrés fisiológico en las colonias, debido al incremento $\mathrm{y}$ mantenimiento de altas temperaturas en la columna de agua (por mas de 12 semanas), así como las subsecuentes afectaciones por enfermedades. Con relación a las poblaciones de D.antillarum, se observaron muchos individuos adultos de la especie entre escombros coralinos y rocas de playa en los fondos someros, sin embargo, no se dispone de información alguna sobre sus densidades.

Entre los parámetros indicadores de la salud de un arrecife se encuentra el porcentaje de cobertura viva que presente (Jackson, 2013). La cobertura promedio de coral vivo estimada para Isla de Aves (22.30\%) se puede considerar dentro de la amplitud de valores que se ha reportado actualmente para el Caribe, los cuales han descendido notablemente en las últimas décadas dado el deterioro que han sufrido los arrecifes (Jackson, 2013). En la zona geográfica donde se encuentra Isla de Aves, uno de los factores que pudiera tener gran impacto en la cobertura de coral vivo son los huracanes y tormentas, ya que en esta área son muy frecuentes estos fenómenos climatológicos (Arteaga, 2002). Aunque no se tienen datos específicos sobre la localidad de estudio, se pueden mencionar casos ocurridos en islas relativamente cercanas a Isla de Aves, como Martinica donde la cobertura promedio de coral vivo pasó de de $37.3 \%$. a $23.2 \%$, luego del Huracan Dean en el año 2007 (Rousseau, Galzin \& Maréchal, 2010) Asimismo, en Dominica, la cobertura, pasó de 17\% (Steiner, 2003) a menos de 5\%
(Arnold et al., 2010) luego de un huracán y Anguilla donde la cobertura descendió de 14\% en 1990 a 4\% en 2009 (Wynne, 2013). En otra isla cercana, Guadalupe, Bouchon et al. (2008) reportaron la destrucción de corales, esponjas y octocorales hasta los $25 \mathrm{~m}$ de profundidad por olas de $13 \mathrm{~m}$ de altitud generadas por los huracanes Luis, Marylin y Lennin, en la década de los 90. Considerando la información recabada por Olivares \& Piñero (2010) en un recuento histórico sobre ciclones tropicales ocurridos en el Océano Atlántico entre 1871 y 2008, se puede tener una idea del impacto que estos fenómenos han generado en la zona a lo largo del tiempo. En dicho compilado se reportan 113 ciclones cercanos a la Isla, 67 de estos huracanes, 36 tormentas tropicales y 10 no clasificados por no presentar datos. Los meses de agosto y septiembre representaron las épocas con mayor porcentaje de ocurrencia de ciclones, afectando principalmente los sectores Sur y Norte de la Isla.

Una característica particularmente importante de Isla de Aves es el bajo impacto antropógenico que tiene, comparado con otras islas del Caribe. En esta isla no hay presión de pesca ni de turismo y el desarrollo de actividades humanas es muy bajo, ya que la población residente en la isla es mínima (15 personas aprox.). La única actividad que se pudiera considerar de impacto es el vertimento de los desechos orgánicos al mar sin planta de tratamiento, situación sobre la que se tomarán medidas próximamente y por otro, lado las grandes cantidades de guano de las aves residentes en la Isla, probablemente tengan un efecto sobre las aguas circundantes en el sector occidental, que debe ser evaluado. Es muy distinta la situación en varias de las Islas cercanas como Guadalupe, Martinica, Antigua y Bermuda, las cuales tienen un gran desarrollo turístico y costero (Smith, Rogers \& Bouchon, 1997). Incluso en Martinica se reportó una invasión de Sargassum probablemente por eutrofización producto de descargas humanas en la década de los años 90, que afectó también a Guadalupe (Bouchon, Bouchon, Louis, 1992). 
Aún se deben seguir las investigaciones sobre las comunidades bentónicas de Isla de Aves, especialmente en las zonas más profundas y se deben hacer evaluaciones en diferentes épocas del año, esto último permitiría evaluar los efectos que puedan generar los cambios en variables ambientales como la temperatura, o la presión atmosférica (relacionada con la frecuencia e intensidad de tormentas y huracanes) en las comunidades de corales y octocorales de la Isla, así como de otras comunidades de invertebrados presentes, factores que posiblemente son los responsables de gran parte del deterioro o perdida coralina en estos fondos. Los resultados de este tipo de estudios en Isla de Aves, no sólo son de gran valor para Venezuela, por las razones anteriormente citadas en este trabajo, sino para las islas cercanas ubicadas al este y al norte de Isla de Aves (sujetos a condiciones ambientales similares), ya que sus arrecifes y comunidades coralinas podrían ser consideradas como "pristinas" con relación al impacto directo de actividades humanas derivadas del uso de la tierra, modificación de las costas, tratamiento de las aguas, turismo desmesurado, pesquerías, u otro tipo de actividad antropogénica contaminante, representando un "blanco", de gran interés comparativo.

\section{AGRADECIMIENTOS}

Este trabajo forma parte del Subproyecto "Caracterización biótica y condición de salud de las comunidades coralinas y arrecifes de Isla de Aves" Coordinado por E.Villamizar, (IZET, UCV). Se encuentra enmarcado dentro del MacroProyecto Institucional del Instituto de Zoología y Ecología Tropical de la UCV, que lleva por título "Evaluación Integral de las Condiciones bióticas y abióticas de Isla de aves: estudio de línea base". Los autores agradecen al personal de la Base Científico Naval Simón Bolívar por toda la colaboración prestada durante la ejecución de las Campañas Científicas, a la Dirección de Hidrografía y Navegación (DHN) por el apoyo logístico y a la Universidad Central de Venezuela por colaborar parcialmente con los viáticos y al Fondo Nacional de Ciencia y Tecnología (FONACIT) por la subvención otorgada para participar en la $36^{\text {ava }}$ edición de los Laboratorios Marinos del Caribe. Agradecemos igualmente a Freddy Bustillos por la elaboración del mapa y a los evaluadores del presente manuscrito, cuyo análisis crítico permitió enriquecerlo.

\section{RESUMEN}

El Refugio de Fauna Silvestre Isla de Aves (RFSIA) representa la porción más septentrional del territorio Venezolano, generando $135000 \mathrm{~km}^{2}$ de Zona Económica Exclusiva. Los estudios sobre sus comunidades coralinas son escasos y en su mayoría datan de la década de los setenta, por su ubicación a $650 \mathrm{~km}$ al nor-este del Puerto de la Guaira y por su condición de zona militar. Para actualizar y obtener una línea base cuantitativa sobre la estructura de las comunidades coralinas de la isla, se estimó la cobertura lineal, estructura de tallas, composición y abundancia de corales y octocorales. Evaluamos 16 sitios alrededor de la isla empleando el Protocolo AGGRA (banda-transectas de $\left.10 \mathrm{~m}^{2}\right)$ entre 1.5 y $21 \mathrm{~m}$ de profundidad ( $\mathrm{n}=67$ transectas) y se realizaron censos visuales en 5 sitios más. Observamos un total de 2327 colonias pertenecientes a cuatro especies de hidrozoos y 36 especies de corales pétreos pertenecientes a 11 familias. Los valores de diversidad, dominancia y equitatividad de la comunidad de corales oscilaron entre 0.78 y $2.12(\mathrm{~S}-\mathrm{W}) ; 0.15$ y 0.61 y 0.57 y 0.92 respectivamente. La mayor parte de los corales presentaron valores de abundancia relativa menores al 3\%, a excepción de Porites astreoides (25.57\%), Pseudodiploria strigosa (18.22\%) y Siderastrea siderea (14.44\%). La estructura de tallas de estas especies estuvo representada en su mayoría por las categorías de tallas más pequeñas, entre $\operatorname{los} 3$ y $30 \mathrm{~cm}$ de diámetro máximo y entre 0 y $5 \mathrm{~cm}$ de altura. Se identificaron un total de 13 especies de octocorales pertenecientes a 3 familias, siendo Pseudopterogorgia americana la especie dominante en abundancia. La cobertura promedio de coral vivo (incluyendo hidrocorales) fue de $22.30 \%$ ( $\mathrm{SE}=1.73$ ) (coral muerto $15.45 \%, \mathrm{SE}=3.28$ ). El coral muerto es la que presentó el mayor porcentaje de disimilaridad entre sitios (9.21\%) (ANOSIM); con un porcentaje de contribución de $16.57 \%$ (Análisis SIMPER). La cobertura viva lineal de octocorales varió entre 0 y $21.35 \%$ ( $\mathrm{SE}=3.65)$ con un promedio de $6.38 \%(\mathrm{SE}=0.99)$. Las investigaciones sobre las comunidades bentónicas de Isla de Aves deben continuar, especialmente en las zonas más profundas, para evaluar las ocndiciones ecológicas.

Palabras claves: corales, octocorales, abundancia, diversidad, Isla de Aves, Venezuela. 


\section{REFERENCIAS}

Almeida, P., \& Goddard. D. (1974). Biología y geología del fondo alrededor de Isla de Aves hasta $10 \mathrm{~m}$ de profundidad - Boletín de la Sociedad Venezolana de Ciencias Naturales La Salle. Tomo, XXXI, 128-129.

Arnold, L., Davis., S., Macedo, D., Morris, J., Sklar, A., \& Stockman, R. (2010). A Comparative Study of Dominican Coral Reef Health and Influencing Factors. Texas: A\&M University.

Aronson, R. B., \& Precht. W.F. (2001). White-band disease and the changing face of Caribbean coral reefs. In The Ecology and Etiology of Newly Emerging Marine Diseases (pp. 25-38). Netherlands: Springer

Arteaga A., C. J. (2002). Isla de Aves. Bastión Venezolano en el Mar Caribe. Dirección de Geografia y Cartografia de las Fuerzas Armadas (DIGECAFA) de Venezuela. Caracas, Venezuela: Fuerte Tiuna.

Ballantine, D. L., Appeldoorn, R. S., Yoshioka, P., Weil, E., Armstrong, R., Garcia, J. R., Otero, E., Pagan, F., Sherman, C., Hernandez-Delgado, E. A., Bruckner, A., \& Lilyestrom, C. (2008). Biology and Ecology of Puerto Rican Coral Reefs, pp. 375-406. In Riegl, B. M. \& Dodge, R. E. (Eds). Coral Reefs of the USA. New York: Springer.

Bak, R. P. M., \& Luckhurst. B. E. (1980). Constancy and change in coral reef habitats along depth gradients at Curacao. Oecologia, 47, 145 - 155.

Bak, R. P. M., \& Meesters. E. H. (1999). Population structure as a response of coral communities to global change. American Zoologist, 39, 56-65.

Bastidas, C., Bone, D., Cróquer, A., Debrot, D., García, E., Humanes, A., Ramos, R. \& Rodríguez, S. (2012). Massive hard coral loss after a severe bleaching event in 2010 at Los Roques, Venezuela. Journal of Tropical Biology, 60, 29-37.

Bastidas , C., Cróquer, A., \& Bone, D. (2006). Shifts of dominance species after a mass mortality on a Caribbean Reef. Proceedings of the $10^{\text {th }}$ International Coral Reef Symposium, 4-1, 989-993.

Bastida-Zavala, J. R., Beltrán-Torres, A. U., GutiérrezAguirre, M. A., \& de la Fuente Betancourt, G. (2000). Evaluación rápida de los arrecifes parche de Majagual, Quintana Roo, México. Revista de Biologia Tropical, 48, 25-37.

Bayer, F. M. (1961). The Shallow- Water Octocorallia of the West Indian Region. Martinus Nijoff, The Hague.

Bayer, F. M., Grasshoff, M., \& Verseveldt. J. (1983). Illustrated Trilingual Glosssary of Morphological and Anatomical Terms applied to Octocorallia. The Netherlands: E.J. Brill, Leiden.

Boadas, H. (2011). Caracterización de las comunidades de corales escleractíneos más representativas de la franja de sotavento de los islotes la Pecha y Puerto Real,
Archipiélago de Los Frailes, Dependencia Federal, Venezuela. Trabajo de Grado para optar a Bióloga Marina. Universidad de Oriente Núcleo Nueva Esparta, Escuela de Ciencias Aplicadas al Mar (ECAM).

Bone, D., Cróquer, A., Klein, D., Perez, D., Losada, F., Martín, A., Bastidas, C., Rada, M., Cruz, J.J., Galindo, L., \& Penchaszadeh, P. (2001). Programa Caricomp: Monitoreo continuo del Parque Nacional Morrocoy. Interciencia, 26, 457-461.

Bouchon, C., Bouchon-Navaro, Y., \& Louis, M. (1992). A first record of a Sargassum (Phaeophyta, algae) outbreak in a Caribbean coral reef ecosystem. Proceedings of the 41st Gulf and Caribbean Fisheries Institute, St.Thomas, USVI, 171-188.

Bouchon C., Portillo, P., Bouchon- Navarro, Y., Max, L., Hoetjes, P., Brathwaite, A., Roach, R., Oxenford, H.,'Farrell, S. O., \& Day, O. (2008). Status of coral reefs of the Lesser Antilles after the 2005 coral bleaching event. En Wilkinson C, Souter D (eds) Status of Caribbean coral reefs after bleaching and hurricanes in 2005. Townsville: GCRMN.

Brownell, W., \& Guzmán, C. (1974). Ecología de la Isla de Aves con especial referencia a los peces. Memoria de la Sociedad de Ciencias Naturales La Salle Nro. 98. Tomo, XXXIV, 91 - 168.

Bruckner, A. W., \& Bruckner, R. J. (2003). Condition of coral reefs off less developed coastlines of Curaçao (part 1: stony corals and algae). Pp 370 - 393. En J.C Lang (Ed) Status of coral reef in a Western Atlantic. Results of initial surveys, Atlantic and Gulf Rapid Assesment (AGRRA). Atoll Research Bulletin 496.

Buccimazza, V. (1984). Corales escleractinios y algunas relaciones ambientales en la Isla de Coche, Venezuela. Trab. Grado Lic. Biología, Universidad de Oriente, Cumaná, Venezuela.

Budd, A. F., Fukami, H., Smith, N. D., \& Knowlton, N. (2012). Taxonomic classification of the reef coral family Mussidae (Cnidaria: Anthozoa: Scleractinia). Zoological Journal of the Linnean Society, 166, 465529. doi: 10.1111/j.1096-3642.2012.00855.x

Bythell, J. C., Gladfelter, E. H., \& Bythell, M. (1993). Chronic and catastrophic natural mortality of three common Caribbean reef corals. Coral Reefs, 12, $143-152$.

Caballero, H., Rosales, D., \& Alcalá, A. (2005). Estudio diagnóstico del arrecife coralino del Rincón de Guanabo, Ciudad de la Habana, Cuba. 1. Corales, gorgonáceos y esponjas. Revista Invest de la Mar, 26(3), 207-217.

Cairns, S. D., Hoeksema, B. W., \& Van Der Land, J. (1999). Appendix: List of Extant Stony Corals. Atoll Research Bulletin, 459, 13-46.

Cairns, S. D., \& Kitahara, M. V. (2012). An illustrated key to the genera and subgenra of the recent 
azooxanthellate Scleractinia (Cnidaria, Anthozoa), wth an attached glossary. ZooKeys, 227, 1-47

Carpenter, R. C. (1990). Mass mortality of Diadema antillarum. I. Long-term effects on sea urchin populationdynamics and coral reef algal communities. Marine Biology, 104, 67-77.

Chornesky, E. A., \& Peters, E. C. (1987). Sexual reproduction and colony grow 1 hin the Scleractinian Porites asteroides. Biological Bulletin Marine Biology Laboratory, Woods Hole, 172, 161-177.

Cróquer, A., \& Bone, D. (2003). La enfermedad de Banda Amarilla: ¿Un nuevo problema en el arrecife de Cayo Sombrero: Parque Nacional Morrocoy? Journal of Tropical Biology, 51(3),167-172.

Cróquer, A., Weil, E., \& Zubillaga, A. L. (2005). Effects of white plague disease-II outbreak on the coral community structure of Madrizquí Key, Los Roques National Park, Venezuela. Caribbean Journal of Science, 41(4), 815-823.

Cróquer, A., Debrot, D., Klein, E., Kurten, M., Rodriguez, S., \& Bastidas, C. (2010). What two years of monitoring tell us about Venezuelan coral reefs? The southern Tropical America node of the Global coral reef monitoring network (STA-GCRMN). Journal of Tropical Biology, 58, (1) 51-65.

Dalongeville, R., \& Sanlaville, P. (1984). Essai de synthèse sur le beach-rock.Le beach-rock. Paris, Travaux de la maison de l'Orient, 8, 41-50.

Del Mónaco, C., Narciso, S., Alfonso, F., Gimenez, E., \& Bustillos, F. (2010) Evaluación de las comunidades de corales y peces de algunos arrecifes de la Isla la Tortuga y cayos adyacentes, Venezuela. Boletín del Centro de Investigaciones Biológicas, 44(3), 353-376.

Domínguez, J. G., Gómez, J. C. Ricaurte, C., Mayo, G., Orejarena, J., Díaz, J. M., \& Andrade, C. A. (2010). Cobertura de los fondos y paisajes bentónicos asociados a formaciones diapíricas en los bancos de Salmedina, Plataforma Continental del Caribe Colombiano. Bological Investigations Marine and Costoas, 39(1), 117-135.

Eizaga, M. (2013). Estructura de las comunidades coralinas de Adicora, estado Falcon, Venezuela. Trabajo Especial de Grado, Universidad Nacional Experimental Francisco de Miranda.

Etnoyer, P. J., Wirshing, H. H., \& Sánchez, J. A. (2010). Rapid Assessment of Octocoral Diversity and Habitat on Saba Bank, Netherlands Antilles. PLoS ONE, 5(5), e10668. doi:10.1371/journal.pone.0010668.

Foster, A. B. (1979). Phenotypic plasticity in the reef corals Montastraea annularis (Ellis \& Solander) and Siderastrea siderea (Ellis \& Solander). Journal of Experimental Marine Biology and Ecology, 39(1), 25-54.
García, A., Cróquer, A., \& Pauls, S.M. (2003). Estado actual de las enfermedades y otros signos de deterioro coralino en siete arrecifes del Parque Nacional Archipiélago Los Roques, Venezuela. Revista de Biologia Tropical, 51(4), 173 - 180.

García-Sais, J. R., Castro, R., Sabater-Clavell, J., Esteves, R., Williams, S., \& Carlo, M. (2009). Monitoring of coral reef communities from natural reserves in Puerto Rico: Isla Desecheo, Isla de Mona, Rincon, Guanica, Ponce, Caja de Muerto and Mayaguez, 2008-2009. Final Report. NOAA GRANT NA07NOS4260055.

Gladfelter, W. B. (1982). White-band disease in Acropora palmata: implications for the structure and growth of shallow reefs. Bulletin of Marine Science, 32(2), 639-643.

Green, D. H., Edmunds, P. J., \& Carpenter, R.C. (2008). Increasing relative abundance of Porites astreoides on Caribbean reefs mediated by an overall decline in coral cover. Marine Ecology Progress Series, 359, $1-10$.

Gremone, C., \& Gómez, J. (1983). Isla de Aves como área de desove de la tortuga verde Chelonia mydas. Biología reproductiva y morfometría con observaciones adicionales sobre el ecosistema y las aves 1979/1983. Fundación de la Defensa de la Naturaleza

Guada, H. J., \& Solé, G. (2000). Plan de Acción para la Recuperación de las Tortugas Marinas de Venezuela. Informe Técnico del PAC No. 39. UNEP Caribbean Environmental Program, Kingston, Jamaica.

Guzmán, H. M., \& Cortés, J. (1985). Organismos de los arrecifes coralinos de Costa Rica. IV. Descripción y distribución geográfica de octocoralarios (Cnidaria: Anthozoa) de la costa del Caribe. Brenesia, 24, 125-173.

Guzmán, H. M., \& Guevara, C. (2001). Arrecifes coralinos de Bocas del Toro, Panamá: IV. Distribución, estructura y estado de conservación de los arrecifes continentales de Península Valiente. Revista de Biologia Tropical, 49, 53-66.

Humann, P., \& Deloach, N. (2002). Reef Coral Identification: Florida, Caribbean, Bahamas. Florida: New World Publications.

Hung, M. F. (1985). Los corales pétreos del Parque Nacional Archipiélago de Los Roques. Tesis, Escuela Biología. Univ. Central de Venezuela, Caracas.

Jackson, J. B. C. (2013). Why are some Caribbean coral reefs much healthier than others?.Proceedings of the 36th Scientific Meeting of the Association of Marine Laboratories of the Caribbean (AMLC) Ocho Rios, Jamaica.

Janes, M. P., \& Wah, L. M. (2005). Results of the International Workshop on the taxonomy of octocorals. March 20-26, 2005. University of Kerala, India. 
Kemp, D. W., Oakley, C. A., Thornhill, D. J., Newcomb, L. A., Schmidt, G. W., \& Fitt, W. K. (2011). Catastrophic mortality on inshore coral reefs of the Florida Keys due to severe low-temperature stress. Global Change Biology, 17(11), 3468-3477.

Kinzie, R. A. (1973). The zonation of West Indian gorgonians. Bulletin of Marine Science, 23(1), 93-155.

LaJeunesse T. (2002). Diversity and community structure of symbiotic dinoflagellates from Caribbean coral reefs. Marine Biology, 141, 387-400

Lessios, H. A. (1988). Mass mortality of Diadema antillarum in the Caribbean: what have we learned? Annual Reviews in Ecological Systematics, 19, 371-393.

Lirman, D., \& Fong, P. (2007). Is proximity to landbased sources of coral stressors an appropriate measure of risk to coral reefs? An example from the Florida Reef Track. Marine Pollution Bulletin, 54, 779-791.

Lirman, D., \& Miller, M. W. (2003). Modeling and Monitoring Tools to Assess Recovery Status and Convergence Rates between Restored and Undisturbed Coral Reef Habitats. Restorative Ecology, 11(4), 448-45.

Miloslavich, P., \& Klein, E. (2005). Caribbean Marine Biodiversity: The known and the unknown. Pennsylvania, USA: DEStech Publications Inc.

Miloslavich, P., Dıaz, J.M., Klein, E., Alvarado, J.J., Dıaz, C., Gobin, J., Escobar-Briones, E., Cruz-Motta, J.J., Weil, E., Cortes, J., Bastidas, A.C., Robertson, R., Zapata, F., Martın, A., Castillo, L., Kazandjian, K. \& Ortiz, M. (2010). Marine Biodiversity in the Caribbean: Regional Estimates and Distribution Patterns. PLoS ONE, 5(8), e11916. doi:10.1371/journal. pone. 0011916 .

Olivares, M.A. (1971). Estudio taxonómico de algunos madreporarios del Golfo de Cariaco, Sucre, Venezuela. Bological Institute of Oceanography, 10, 73-78.

Olivares, L., \& Piñero, A. (2010). Los ciclones tropicales en las costas de Venezuela e Isla de Aves. Período 1851-2008. Tecnocolor.

Pantin, H. J. (1972). Nuevas Observaciones Geológicas acerca de la Isla de Aves. Memorias de la VI Conferencia Geologica del Caribe.

Pauls, S.M. (1982). Estructura de las comunidades coralinas de la Bahía Mochima, Venezuela. MSc. Tesis, Univ. De Oriente, Cumaná.

Pauls, S. (1995). Inventario de fauna (Porifera, Cnidaria y Echinodermata) y caracterizacion de comunidades marinas de algunos cayos del Parque Nacional Morrocoy. En: Klein, E \& D. Bone (eds.). Ecosistema Morrocoy: estado actual de las investigaciones $y$ perspectivas futuras. INTECMAR, Universidad Simon Bolivar.

Pérez Nieto, H. (1990). Posibles medidas de protección fisica de la Isla de Aves e Impacto de las mismas.
Seminario-Taller sobre la Isla de Aves. Caracas, Venezuela.

Phelps, Jr . W. H. (1945). Las Aves de las Islas los Testigos, los Frailes y la Tortuga. Boletín de la Sociedad Venezolana de Ciencias Naturales, 60, 257-283.

Ramírez-Villaroel, P. (2001). Corales de Venezuela. Consejo de Investigación de la Universidad de Oriente, Fundaconferry, Comisión de Investigación del Núcleo de Nueva Esparta de la Universidad de Oriente, Porlamar.

Rogers, C.S. (1983). Sublethal and lethal effects of sediments applied to common Caribbean Reef corals in the field. Marine Pollution Bulletin, 14(10), 378-382.

Rousseau, Y., Galzin, R., \& Maréchal, J. P. (2010). Impact of hurricane Dean on coral reef benthic and fish structure of Martinique, French West Indies. Cybium, 34(3), 243-256.

Sant, S., Prieto, A., \& Méndez, E. (2002). Composición de especies de corales (Scleractinia y Milleporina) en Manzanillo, Golfo de Santa Fe, Parque Nacional Mochima, Venezuela Saber, Universidad de Oriente, Venezuela, 14(2), 89-93.

Smith, A. H., Rogers, C. S., \& Bouchon, C. (1997). Status of western Atlantic coral reefs in the Lesser Antilles. Proceedings of the 8th Int. Coral Reef Symposium, 1, 351-356.

Steiner, S. C. C. (2003). Stony Corals and Reefs of Dominica (Lesser Antilles). Atoll Research Bulletin, 498, ITME Research Reports 14.

Steiner, S. C. C., \& Kerr. J. M. (2007). Stony corals in Dominica during the 2005 bleaching episode and one year later. Revista de BiologÃa Tropical, 56.

Stemann, T. A. (1991). Evolution of the reef coral family Agariciidae (Anthozoa: Scleractinia) in the Neogene through Recent of the Caribbean. Ph.D dissertation. Univ. of Iowa, Iowa, EEUU.

Sullivan, K. M., Chiappone, M., \& Lott, C. (1994). Abundance patterns of stony corals on plataform margin reef of the Caicos Bank. Bahamas Journal of Science, 1(3), 1-12.

Szmant, A. M., \& Gassman, N. J. (1990). The effects of prolonged "bleaching" on the tissue biomass and reproduction of the reef coral Montastraea annularis. Coral Reefs, 8, 217-224.

Urich, J. F. (1977). Estructura comunitaria de un arrecife coralino al suroeste de la Orchila, Venezuela. Tesis, Escuela Biología. Univ. Central de Venezuela, Caracas.

Valdivia, A., \& De la Guardia, E. (2004). Estructura de la comunidad de corales en el arrecife costero de Boca de Canasí, La Habana, Cuba. Revista Investigacion de la Mar, 25(1), 15-22. 
Vandel, E., Pichon, M., \& Joannot, P. (2012). Taxonomic inventory of Scleractinia in French overseas territories. Proceedings of the 12th International Coral Reef Symposium, Cairns, Australia,

Villamizar, E. (2000). Estructura de una comunidad arrecifal en Falcón, Venezuela, antes y después de una mortandad masiva. Revista de Biologia Tropical, 47,19-30.

Villamizar, E., Posada, J., \& Gómez, S. (2003). Rapid assessment of coral reefs in the Archipiélago de los Roques National Park, Venezuela (part 1: stony corals and algae). In: J.C. Lang (ed.), Status of Coral Reefs in the western Atlantic: Results of initial Surveys, Atlantic and Gulf Rapid Reef Assessment (AGRRA) Program. Atoll Research Bulletin, 496, 512-529.

Villamizar, E., Camissotti, H., Rodríguez, B., Pérez, J., \& Romero, M. (2008). Impacts of the 2005 Caribbean Bleaching Event at Archipiélago de Los Roques, National Park, Venezuela. Revista de Biologia Tropical, 56, 255-270.
Weil, E. (1985). Lista de los corales escleractínidos de aguas someras del Parque Nacional Archipiélago de Los Roques. Informe Técnico de la Fundación Científica Los Roques.

Weil, E. (2003). Coral and coral reefs of Venezuela, pp. 303-330. En Cortés, J. (ed). Latin American Coral Reefs. Amsterdam: Elsevier.

Weil, E. (2006). Diversidad y abundancia relativa de corales, octocorales y esponjas en el Parque Nacional Jaragua, República Dominicana. Revista de Biologia Tropical, 54(2), 423-443.

Wells, J. W. (1973). New and old scleractinian corals from Jamaica. Corals from Jamaica. Bulletin of Marine Science, 23(1), 16-58.

Wynne, S. P. (2013). Coral Reefs of Anguilla, pp. 13-22. En C.R.C. Sheppard, C. R. C. (ed). Coral Reefs of the United Kingdom Overseas Territories. Netherlands: Springer. 